\title{
Decoding the single-cell landscape and intercellular crosstalk in the transplanted liver: a
}

\section{4-dimension mouse model}

Haitao Huang ${ }^{1,2}$, Xueyou Zhang ${ }^{1,2}$, Hui Chen ${ }^{2}$, Shi Feng ${ }^{3}$, Cheng Zhang ${ }^{1,2}$, Ruihan Chen ${ }^{1,2}$, Yimou Lin $^{1,2}$, Qinghua Ji ${ }^{4}$, Qi Ling ${ }^{1,2,5^{*}}$.

${ }^{1}$ Department of Surgery, the First Affiliated Hospital, Zhejiang University School of Medicine, Hangzhou, Zhejiang, China.

${ }^{2}$ NHC Key Laboratory of Combined Multi-Organ Transplantation, Hangzhou, Zhejiang, China.

${ }^{3}$ Department of pathology, the First Affiliated Hospital, Zhejiang University School of Medicine, Hangzhou, Zhejiang, China.

${ }^{4}$ Zhejiang Puluoting Health Technology Company Limited, China.

${ }^{5}$ Collaborative innovation center for diagnosis and treatment of infectious diseases, China.

\section{Correspondence author emails}

${ }^{*}$ Correspondence and request for materials should be addressed to Qi Ling (email: lingqi@zju.edu.cn)

\section{Key words}

liver transplantation; graft remodeling; crosstalk; single-cell RNA sequencing; cytometry by time-of-flight

\section{Abbreviations}

LT, liver transplantation; scRNA-seq, single-cell RNA sequencing; CyTOF, cytometry by time-of-flight; ALT, alanine aminotransferase; AST, aspartate aminotransferase; IRI, liver ischemia-reperfusion injury; H\&E, hematoxylin and eosin; TUNEL, terminal deoxynucleotidyl transferase-mediated dUTP nick-end labeling; tSNE, t-distributed stochastic neighbor embedding; GO, Gene Ontology; GSVA, gene set variation analysis; PCA, principal component analysis; NK, natural killer; DCs, dendritic cells; DEGs, differentially expressed genes; GDCs, graft-derived cells; RDCs, recipient-derived cells 


\section{ABSTRACT}

Graft remodeling after transplantation maintains graft functionality and determines graft survival. However, a comprehensive understanding of cellular diversity and interplay during graft remodeling remains to be fully characterized. In this study, we established a well tolerant C57BL/6 to C57BL/6 orthotopic liver transplantation (LT) mice model and observed two stages of graft recovery including an acute phase and a steady phase. We next performed single-cell RNA sequencing (scRNA-seq) and cytometry by time-of-flight (CyTOF) and recorded the cellular hierarchy in the transplanted liver during the two stages. Besides the dynamic change of cell proportion, it was notable that recipient-derived cells took over the transplanted liver in most cell types (e.g., B cells, T cells, dendritic cells, granulocytes and monocytes) except $\mathrm{CD}_{206}{ }^{+} \mathrm{MerTK}^{+}$macrophages and $\mathrm{CD} 161^{+}$ $\mathrm{CD} 49 \mathrm{a}^{+} \mathrm{CD} 49 \mathrm{~b}$ natural killer cells. We then focused on macrophages and captured 5 distinct transcriptional signatures to define novel subclusters. Using a ligand-receptor interaction strategy, we identified specific macrophage-hepatocyte interactions during the acute and stable phases, causing metabolic remodeling in the transplanted liver. Our results delineated a 4-dimension cell atlas (type-proportion-source-time) of the transplanted liver, which sheds light on the physiological process of liver graft maintenance and graft-recipient crosstalk. 


\section{INTRODUCTION}

Liver transplantation (LT) is the most effective salvage treatment for various end-stage liver diseases [1]. With the development of the transplant technique and immunosuppressant in the last decades, LT has become a routine surgical procedure with a high success rate [2]. Long-term survival and improved quality of life are the current purposes after LT [3, 4]. In liver recipients, long-term graft survival mostly relies on the prompt recovery of graft function and avoidance of post-transplant complications such as graft dysfunction, rejection, biliary complications and metabolic disorders [5-7].

Graft remodeling has been considered a complex and well-orchestrated process that involves the cellular composition and functional state from multiple lineages [8]. In LT, immune cells derived from the recipient's peripheral blood invade through the hepatic sinusoid and move towards the interstitial space, where interplay with graft-derived cells to further reorganize immune and metabolic homeostasis $[9,10]$. During the early post-LT period, parameters reflecting liver injury and inflammatory response would be predictors for prognosis. For example, alanine aminotransferase (ALT) and $\gamma$-GT levels on postoperative day 1 have been considered as independent predictors of early allograft dysfunction [11]. A combined detection of IL-10, IL-17, and CXCL10 hold an excellent prediction property for acute rejection [12]. Therefore, a deep understanding of graft remodeling including the recipient-graft interplay is essential to promote the recovery of the transplanted liver and prevent post-transplant complications.

The single-cell approach has emerged as a powerful tool to deconstruct the composition and function of complex tissues at single-cell resolution [13]. Recently, single-cell RNA sequencing (scRNA-seq) has been applied to dissect the complex landscapes of organogenesis, including liver development [14] and decidualization [15]. The advent of the high-throughput single-cell profiling technology provides a foundation for "transplanted liver atlas". In this study, we employed scRNA-seq and cytometry by time-of-flight (CyTOF) to comprehensively reveal the cell states and sources involved in liver graft remodeling. We then uncovered an interplay between hepatocytes and macrophages using ligand-receptor interactions based on the transcriptome data [16]. Our analyses constructed a detailed molecular and cellular map and revealed complex intercellular crosstalk in the transplanted liver.

\section{MATERIALS AND METHODS}

\section{Animals treatments}

Male 8-12 weeks old wild-type C57BL/6 mice and C57BL/6-Tg (CAG-EGFP) mice were purchased from Shanghai Model Organisms Center. All mice were housed at 20 $\square$ with a 12:12 light-dark cycle in a specific pathogen-free animal facility and fed ad libitum with a normal chow diet. All animal experimental protocols were approved by the Institutional Animal Care and Use Committee and the Ethics Committee of Zhejiang University. Livers from C57BL/6-Tg (CAG-EGFP) mice (all tissue 
expressing eGFP) used as donors and wild-type C57BL/6 mice were used as recipients for the orthotopic LT model. Surgery was performed as we described before [17]. After the postoperative restoration of temperature and rehydration, mice were sent to the individually ventilated cages for housing. The transplanted liver and serum samples were collected at 3H, 6H,12H, 1D, 3D, 7D, and 14D post-LT. Biochemical parameters including ALT and aspartate aminotransferase (AST) were measured with a BioVision system. The severity of liver ischemia-reperfusion injury (IRI) was graded by modified Suzuki's histological criteria [18].

\section{Immunohistochemistry}

Immunohistochemistry was performed as we described previously [19]. Liver specimens $(4 \mu \mathrm{m})$ were stained with hematoxylin and eosin (H\&E). Two independent pathologists reviewed and confirmed the pathohistological features of all the samples. For staining of neutrophils and macrophages, anti-Ly6g (Abcam, USA, ab238132) and anti-F4/80 (Bio-rad, USA, MCA497R) were used. For terminal deoxynucleotidyl transferase-mediated dUTP nick-end labeling (TUNEL) staining, liver specimens were stained using the In Situ Cell Death Detection Kit (Roche, USA, 11684817910) according to the manufacturer's protocol. Labeled cells were counted with 5 randomly selected $400 \times$ fields from each sample.

\section{Quantitative real-time PCR}

Total RNA was extracted using TRIzol reagent (Invitrogen, USA, 15596018) and quantitative real-time PCR was performed using the HiScript II One Step qRT-PCR SYBR Green Kit (Vazyme Biotech, China, Q221-01) in an Applied Biosystems QuantStudio 5 Real-Time PCR Instrument (Applied Biosystem, USA) as we described previously [17]. The detailed primer information was provided in Table $\mathrm{S} 1$.

\section{Tissue dissociation and preparation}

Liver tissues were obtained from pre- and post-LT period. The tissues were transferred into sterile RNase-free culture dishes containing an appropriate amount of calcium-free and magnesium-free PBS on ice and cut into $0.5 \mathrm{~mm}^{3}$ pieces. Then the tissues were washed with PBS and dissociated into single cells in dissociation solution ( $0.35 \%$ collagenase IV, $2 \mathrm{mg} / \mathrm{ml}$ papain, 120 Units/ml DNase I) in $37 \square$ water baths with shaking at $100 \mathrm{rpm}$ for $20 \mathrm{~min}$. Digestion was terminated by PBS containing $10 \%$ fetal bovine serum (V/V). The suspensions were passed through $100 \mu \mathrm{m}$ cell sieves (Corning, Japan, 431752) and then $40 \mu \mathrm{m}$ cell sieves (Corning, Japan, 431750). The flow-through was centrifuged and resuspended in $5 \mathrm{ml}$ of red blood cell lysis buffer (Invitrogen, USA, 00-4300) for $10 \mathrm{~min}$. After incubation, the suspension was centrifuged at $300 \mathrm{~g}$ for $5 \mathrm{~min}$ at $4 \square$. For scRNA-seq, the overall cell viability was confirmed by trypan blue (over $85 \%$ ), single-cell suspensions were counted using a Countess II Automated Cell Counter (Invitrogen, USA, 00-4300), and concentration adjusted to 700-1200 cells/ $\mu 1$.

\section{CyTOF}

Liver single-cell suspensions were processed for CyTOF analysis according to the standard protocol 
as we described previously [17]. Briefly, the single-cell suspensions were labeled by $194 \mathrm{Pt}$ for $5 \mathrm{~min}$ to distinguish live or dead and incubated with Fc blocking mix for 20 min at room temperature to block Fc receptors. Then the single-cell suspensions were stained for $30 \square \mathrm{min}$ with an antibody mix panel (Table S2), followed by DNA Intercalator-Ir overnight staining. Intracellular markers were stained using an intracellular antibody mix for $30 \mathrm{~min}$. Finally, the single cells were washed with PBS, and the signals were detected using a CyTOF system (Fluidigm, USA). The types of immune cells were identified via t-distributed stochastic neighbor embedding (tSNE), followed by density clustering [20]

\section{scRNA-seq}

Liver single-cell suspensions were loaded into the 10x Chromium Controller, and the 10x Genomics Chromium Single Cell 3' kit (V3) was applied according to the manufacturer's protocol. Libraries were sequenced on an Illumina NovaSeq 6000 sequencing system. We used FastQC to perform basic statistics on the quality of the raw reads. Raw reads were demultiplexed and mapped to the reference genome by $10 x$ Genomics Cell Ranger pipeline (https://support.10xgenomics.com/single-cell-geneexpression/software/pipelines/latest/what-is-cell-ra nger). All downstream single-cell analyses were performed using Cell Ranger (version 3.1.0) and Seurat (version 3.2.0) unless mentioned specifically. In brief, for each gene and each cell barcode (filtered by CellRanger), unique molecule identifiers were counted to construct digital expression matrices. Secondary filtration by Seurat: A gene with expression in more than 3 cells was considered as expressed, and each cell was required to have at least 200 expressed genes. We removed low-quality cells with $>20 \%$ mitochondrial RNA content and included cells with $>200$ and $<6000$ genes expressed per cell. Seurat was used to normalizing data, dimensionality reduction, clustering and differential expression analysis. To eliminate batch effects, a reference Seurat object was generated by identifying anchor genes between samples as described previously [14]. For clustering, highly variable genes were selected and the principal components based on those genes were used to build a graph. The tSNE analysis was used for dimension reduction and visualization of gene expression. For differential expression analysis, marker genes for each cluster were determined by comparing the cells of each cluster to all other cells using the FindAllMarkers function in Seurat. For all comparisons between groups and clusters, only genes expressed by at least $10 \%$ of cells were included. Gene Ontology (GO) enrichment analysis of marker genes was implemented by the clusterProfiler R package (version 3.16.1), in which gene length bias was corrected. GO terms with corrected $P$ value less than 0.05 were considered significantly enriched by marker genes. gene set variation analysis (GSVA) analysis of distinct clusters was performed by the GSVA R package (version 1.36.2)[21].

\section{Cell-cell interplay analysis}

To enable a systematic analysis of cell-cell communication, we applied nichenetr package (version 1.0.0) to predict cell-cell interplay as previously reported [22]. NicheNet could predict ligands of 
sender cells that are the most active in affecting gene expression of receiver cells, through the correlation of ligand activity with genes characterized as targets in a prior model that integrates existing knowledge on ligand-to-target signaling path [16]. Using macrophages and hepatocytes subsets, we applied NicheNet to predict ligands that might influence transcription during hepatocytes metabolic remodeling. In the integration analysis, hepatocytes were defined as the receiver population and all macrophage subclusters were defined as potential sender cells. In separate analyses, target macrophage subclusters were defined as potential sender cells. Gene sets of interest were defined based on metabolic-related genes in MSigDB gene sets, including regulation of glucose metabolic process, insulin signaling pathway and lipid homeostasis (Table.S3). A background gene set includes all other genes expressed in at least $10 \%$ of cells in hepatocytes. Activities of ligands were ranked by calculating Pearson correlation coefficients of the ligand-target regulatory potential scores for each ligand and the target indicator vector, which defines a gene as present or absent within the gene set of interest. The circlize $\mathrm{R}$ package (version 0.4.10) was used to represent the regulatory networks between the predicted ligands in distinct macrophage subclusters and their receptors and targets expressed in hepatocytes. Cytoscape (version 3.8.0) was used to depict the regulatory networks between the predicted ligands in the target macrophage subclusters and their targets expressed in hepatocytes.

\section{RESULTS}

\section{Liver graft recovery following transplantation}

Liver graft injury is inevitable due to multiple factors including IRI and surgical stress. To monitor the liver graft recovery after transplantation, we established an orthotopic LT mice model and collected serum and liver tissue samples during the perioperative period (Pre-LT, 3H, 6H, 12H, 1D, 3D, 7D and 14D post-LT; $n=3$ /group, Fig.1A). We observed sharply increased ALT and AST levels at $3 \mathrm{H}$ post-LT. From $3 \mathrm{H}$ to $1 \mathrm{D}$, serum ALT and AST levels gradually decreased and maintained steady after 1D (Fig.1B). We next assessed the morphology organization of transplanted livers using histopathology methods. H\&E staining showed the destruction of hepatic architecture as presented by sinusoidal congestion, hepatocyte necrosis, and ballooning degeneration. Suzuki's histological grading of hepatocellular damage displayed the highest score at 1D (Fig.1C). We further found consistent results in hepatocellular necrosis/apoptosis assessment (Fig.1D). Moreover, we assessed inflammatory cell infiltration using immunohistology and observed that neutrophils and macrophages maintained at high levels during the first day and reduced thereafter (Fig.1E). The cytokine and endothelial cell activation marker expression patterns showed a similar trend with inflammatory cells (Fig.1F). The results suggested an acute injury of graft function within 1D (acute phase) and a steady recovery of graft function after $3 \mathrm{D}$ (stable phase).

\section{Mapping cellular hierarchy in the transplanted liver}


To map the cellular diversity and the dynamic change during the perioperative period, we generated scRNA-seq profiles from 6 liver samples (pre-LT, 3H, 6H, 12H, 3D and 7D post-LT) using 10x Genomics sequencing (Fig.2A). We obtained a total of 22,119 single-cell transcriptomes for analysis. We conducted gene expression normalization, principal component analysis (PCA), and tSNE. Finally, all liver cells could be assigned to 10 distinct types based on marker gene expression and scMCA (http://bis.zju.edu.cn/MCA/blast.html). They were granulocyte (38.8\%, marked with $L y 6 g$ ), T cells (14.5\%, marked with $C d 3 d$ ), macrophages (13.1\%, marked with Adgre1), endothelial cells (9.4\%, marked with Bmp2), B cells (8.3\%, marked with $C d 79 a$ ), dendritic cells (DCs, 6.4\%, marked with Itgax), natural killer (NK) cells (5.9\%, marked with Klrblc), hepatocytes (2.1\%, marked with Apoal), biliary epithelial cells (1.0\%, marked with $\mathrm{Krt19}$ ) and fibroblast (0.4\%, marked with Acta2, Fig. 2B). The differentially expressed genes (DEGs) and GO enrichment analysis further confirmed the cell identity (Fig. 2C and Fig.S1A). Moreover, we observed a significant variation in the proportion of immune cells during the perioperative period (Fig.2D). For instance, granulocytes increased rapidly from $8.1 \%$ to $78.3 \%$ at $6 \mathrm{H}$ post-LT and macrophages increased from $9.7 \%$ to $29.7 \%$ at $12 \mathrm{H}$ post-LT, indicating an IRI-induced inflammatory response. Both T cells and B cells dropped to less than 15\% after $6 \mathrm{H}$ post-LT, suggesting a tolerance in our model. (Fig.2D and Fig.S1B).

\section{The cellular source in the transplanted liver}

To explore the comprehensive graft-recipient interaction following LT, we used an eGFP-labeled donor mouse to distinguish graft-derived cells from recipient-derived cells. The efficiency of eGFP fluorescence was $90 \%$ according our previous report [17]. We acquired liver tissue samples at pre-LT, 12H, 1D, 3D, 7D and 14D post-LT and purified CD45 liver nonparenchymal cells for CyTOF analysis (Fig. 3A), which comprises 42 antibodies. Cells were initially gated and t-SNE visualized to distinct populations. A schematic clustering explanation is depicted in Fig. 3B. Among the 29 clusters, No. 5 could not be categorized and the other 28 were defined as 7 cell types including B cells (2 clusters, marked with CD19), T cells (5 clusters, marked with CD3), DCs (5 clusters, marked with CD11c), NK cells (3 clusters, marked with NKp46 or CD49b), macrophages (8 clusters, marked with F4/80), granulocytes (2 clusters, marked with LY6G) and monocytes (3 clusters, marked with LY6C; Fig. 3C). We observed that graft-derived cells (GDCs) dramatically decreased from $100 \%$ to $11 \%$, whereas the recipient-derived cells (RDCs) sharply increased from $0 \%$ to $89 \%$ within the first day post-LT (Fig. 3D). The cell source maintained a steady level from 1D to 14D. RDCs accounted for most cells at 14D post-LT (26/29 clusters) (Fig. 3E). Of note, two M2 macrophage clusters (cluster 1 and cluster $23 ; \mathrm{F} 4 / 80^{+} \mathrm{CD}_{206}{ }^{+} \mathrm{MerTK}^{+}$) and one $\mathrm{NK}$ cell cluster (cluster $16 ; \mathrm{CD} 61^{+} \mathrm{CD}_{\left.49 \mathrm{a}^{+} \mathrm{CD} 49 \mathrm{~b}\right)}$ ) were composed of around half GDCs at the stable phase (Fig. 3F).

\section{Metabolic remodeling in the transplanted liver}

Considering the specific source and vital role of the macrophage in hepatic homeostasis particularly 
glucose and lipid metabolism, we focused on the metabolic remodeling by analyzing the macrophage-hepatocyte interplay. First, we re-analyzed the scRNA-seq data of macrophages and stratified them into 5 subclusters with distinct transcriptomic signatures (Fig. 4A). We identified the function of different subclusters using GSVA, which showed that subclusters $0,1,2,3$ and 4 were associated with pro-inflammation, ribosome and proteasome, metabolism, immune regulation and allograft rejection, respectively (Fig. 4B). We found that subcluster 0 and 1 expressed high levels of $L y 6 c 2$ and $C c r 2$ (immature monocyte-derived macrophages genes), while subcluster 3 expressed low level $C c r 2$ and high level of $C x 3 c r 1$ (mature monocyte-derived macrophages genes). Subcluster 2 expressed a certain number of Adgre1, Clec4f and Timd4 (Kupffer cells genes). Moreover, we assumed that subcluster 0 and 1 belonged to the M1 phenotype due to the pro-inflammatory markers such as $\operatorname{Tnf}, \mathrm{Ccl} 2$ and $\mathrm{Ccl} 9$, while subcluster 2 belonged to the M2 phenotype due to the prominent expression of M2 markers such as Mrc1 (coding CD206), Cd163 and Mertk (Fig. 4C). We observed the dynamic change of different subclusters in the acute and stable phases. For instance, subcluster 0 sharply increased in the acute phase and decreased in the stable phase, whereas subcluster 2 dramatically decreased in the acute phase and partially recovered in the stable phase (Fig. 4D).

We should also note that subcluster 2 was comprised of both graft- and recipient-derived cells (F4/80 ${ }^{+} \mathrm{CD}_{206^{+}}$MerTK $^{+}$Macrophages) according to the CyTOF findings. In subcluster 2, the genes involved in anti-fibrosis and metabolism such as Cd5l [23], Timd4 [24], Clec2 [25] and Pltp [26] were significantly up-regulated and the genes related to inflammation such as $T n f, I l 1 b$ and $C x c l 2$ [27] were significantly down-regulated compared to other macrophages (Fig. 4E). Furthermore, a comparison of subcluster 2 in the acute and stable phases with that at pre-LT revealed distinct transcriptional programs (Fig. 4F). Compared with pre-LT, genes highly expressed in subcluster 2 in the acute phase were enriched in cytokine receptors and extracellular vesicles. In the stable phase, highly expressed genes were mainly enriched in cytoplasmic translation and ribosome.

Second, we analyzed the metabolic activity of hepatocytes using GSVA and found decreased gluconeogenesis, insulin signaling and lipid metabolic process whereas increased glycolysis in the acute phase. The changed glucose and lipid metabolism well recovered in the stable phase (Fig.5A). Next, we performed NicheNet, which could show ligand-receptor interactions and ligand-to-target signaling paths [16], to analyze the macrophage-hepatocyte interplay. A circle plot was designed to show the interaction between the receptors and target genes in hepatocytes and the most active ligands in macrophages (Fig.5B). Macrophage subcluster 0, 1, 2 and 4 presented potential specific ligands that regulate insulin signal, glucose and lipid metabolic genes in hepatocytes. For example, Ceacaml, Cxcl2, Tnf and Itgam were most expressed in subcluster 0, while Cxcl13, Cxcl16, Apoe and Nampt were expressed in subcluster 2.

Based on the abundant crosstalk between subcluster $0 / 2$ and hepatocytes, we isolated the 3 populations and further predicted the specific ligand-receptor interactions. Ligands activity analysis showed the top 10 ligands in subcluster 0 and 2 (Fig.5C). For instance, Tnf, coding cytokine involved 
in hepatocyte cell death as well as insulin resistance and lipid accumulation [28, 29], was mainly expressed by subcluster 0 . The expression of Tnf increased significantly in the acute phase and recovered in the stable phase (Fig.5D). In addition, hepatocytes strongly expressed Tnf receptor genes tnfrsfla and tnfrsflb in the acute phase, suggesting an enhanced Tnf-Tnf receptor interaction in the acute phase (Fig.5E). Target genes prediction showed that Tnf could regulate different types of metabolic genes, including glucose metabolism genes (Sirt1, Ppara, Akt1, etc.), lipid metabolism genes (Cebpa, Nrlh2, Apoal, etc.) and insulin pathway genes (Pck1, Socs3, Ptprt1, etc.; Fig.5F). For another example, Apoe, coding apolipoprotein E that involved in lipid and lipoprotein metabolism, was mainly expressed by subcluster 2. The expression of Apoe and Apoe receptor genes, such as Sorl1 and $L d l r$, decreased in the acute phase and increased in the stable phase, suggesting an enhanced Apoe-Apoe receptor interaction in the stable phase. Furthermore, target genes prediction showed that Apoe mainly regulated lipid metabolism-related genes in hepatocytes such as Srebf1, Abcal, Apoal, Apoa2, Apob, Igfl, Ldlr, etc. Other ligands such as Ceacam1, Rtn4, C3, Cxcl2, Cxcl13, Timp1, Calm1 and $T g f b 1$, were also predicted to regulate a series of metabolic genes in hepatocytes and ultimately contribute to the changes in metabolism-related genes during the perioperative period.

\section{DISCUSSION}

The occurrence of primary nonfunction, graft dysfunction or rejection dramatically reduced both graft and recipient survival. Therefore, graft recovery during the 7-10 days after LT greatly determines the outcome [30]. In this study, we established a tolerant mice model to understand the physiological process of graft recovery and graft-recipient interaction during the early post-LT period. According to the dynamically detected biochemical and pathological parameters, we proposed a two-phase theory of graft recovery following LT, which includes an acute phase and a stable phase. Acute phase was represented by sharply increased hepatocyte damage and inflammatory cell infiltration, occurring at very early stage typically within $24 \mathrm{~h}$ following LT. The emergence of the peaks of aminotransferases in our model was consistent with most of previous studies [18, 31]. Also, the graft injury would be affected by multiple factors such as ischemia time and perfusate, causing diversity in graft recovery $[32,33]$. Stable phase showed a well recovered liver function after the acute graft injury, which probably started from 3D after LT. Then we conducted a single cell technology for the in-depth understanding of graft remodeling in both acute and stable phases.

We described the transcriptomic landscape of the transplanted liver and depicted the cellular atlas, including the cell types and proportion. The diversity of liver parenchymal and non-parenchymal populations was consistent with previous studies both in mice [34] and human [21]. Given the distinct cell types, we found that the cellular composition of the transplanted liver has changed dramatically during the perioperative period. The persistently elevated inflammatory cells (granulocytes and macrophages) in the transplanted liver throughout the acute and stable phases revealed an IRI-induced inflammatory response, which has been associated with the development of post-transplant 
complications such as tumor recurrence, diabetes, hyperlipidemia and fibrosis [35]. In addition, the transplanted liver in the stable phase had decreased proportions of $\mathrm{T}$ cell and B cell, which were known to promote rejection [36], indicating tolerance in the stable phase.

We next focused on the graft-recipient source in distinct cell types. Our previous pioneering study using the same strategy and focusing on the monocyte-derived cells has revealed that RDCs took over the majority of cells, including monocytes, DCs, M0 and M1 macrophages. This study further explored the cellular source of various nonparenchymal (e.g., macrophages, NK cells, DCs, endothelial cells, $\mathrm{T}$ cells and B cells) in the transplanted liver. The results were consistent with our previous findings that GDCs disappeared in a short time and RDCs made the major contribution to Kupffer cells pool. The result challenged the classical theory that Kupffer cells are locally proliferate and self-renew [37] at least under the circumstance of LT. Furthermore, not only the monocytes-derived cells, most nonparenchymal cells were refreshed by RDCs at steady phase, suggesting a pivotal role of graft-recipient crosstalk during graft remolding. It was very interesting to find that GDCs were still accounted for a half of proportion in certain cell populations including

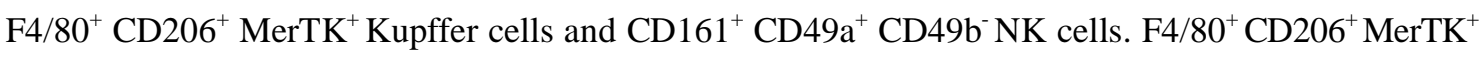
Kupffer cells have been associated with the resolution of inflammation and the compromise repair after ischemia-reperfusion [38, 39], as well as the improved hepatic insulin sensitivity [40]. However, these macrophages with an anti-inflammatory and reparative phenotype have been revealed to drive hepatocellular carcinoma progression and metastasis [37]. In addition, a recent study on CD49a ${ }^{+}$ liver-resident NK have showed that a role for $\mathrm{CD} 49 \mathrm{a}^{+} \mathrm{NK}$ cells in the negative regulation of immune responses and the development of hepatocellular carcinoma[41]. Investigation of diseases associated with Kupffer cells and NK cells after LT (e.g., liver fibrosis [42], rejection[43] and hepatocellular carcinoma [44]) should consider both the graft and recipient genetic backgrounds.

Our previous studies in both large human cohorts [45, 46] and mice models [19] demonstrated an imbalanced hepatic metabolic homeostasis after LT. This study not only proved that hepatocytes showed a metabolic reprogram after LT including insulin resistance, glucose and lipid metabolic homeostasis, but also revealed a comprehensive interplay between macrophage and hepatocytes in the transplanted liver. In previous studies, scRNA-seq analysis has been used to elucidate the diversity of macrophages in human and mouse livers [47, 48]. In this study, we defined 5 macrophage subclusters in the transplanted liver and preliminarily explored their functional transformation at different phases, such as subcluster 0 with high pro-inflammatory activity in the acute phase and subcluster 4 with suppressed rejection activity in the stable phase. Further exploring the interplay between liver macrophages and hepatocytes, macrophages subcluster 0 and subcluster 2 hold the most abundant ligand-receptor pairs and excellent regulatory potential, suggesting an effectively regulation in the metabolic remodeling. For instance, the Tnf-Tnf receptor pair in the acute phase was significantly enhanced, which have been found to be crucial for the development of metabolic disorders [49]. In addition, an enhanced Apoe-Apoe receptor pair, promoting the clearance of remnants of 
triglyceride-rich lipoproteins[50], was found to contribute to the stable phase. Our scRNA-seq data and functional analysis showed that the metabolic remodeling of the transplanted liver was a very complex process, involving a regulatory network of a series of ligands and receptors between macrophages and hepatocytes.

The study had both advantages and limitations. First, to the best of our knowledge, this is the first study to map a 4-dimension cell atlas of the transplanted liver based on a well tolerant LT mice model. The open source website construction is in progress and the datasets would be available for further analyses such as the comparison with the rejection model or marginal graft model. Second, our finding shed a light on the intrahepatic donor-recipient crosstalk, which is the key to understand liver transplant immunology and pathophysiology. For instance, the F4/80 ${ }^{+} \mathrm{CD} 206^{+} \mathrm{MerTK}^{+}$hepatic macrophages had both donor- and recipient-sources. Since this subclass involves in liver fibrosis, nonalcoholic steatohepatitis and tumor recurrence, both donor and recipient genetic background potentially affect the long-term survival of recipients [37, 51]. The predicted ligand-receptor interactions need to be further proved and more cellular interplays need to be explored.

\section{FUNDING}

This study was supported by the National Natural Science Foundation of China (No. 81771713 and 82011530442).

\section{DISCLOSURE}

Q.J. is a member of PLT Tech Inc.

\section{AUTHOR CONTRIBUTIONS}

Q.L. designed the research; H.H., S.F., T.R., and H.C. performed the research; H.H., X.Z and Q.J. analyzed the data; H.H. and R.C. wrote the manuscript; Q.L. reviewed the manuscript.

\section{REFERENCE}

1. Mathur, A.K. and J. Talwalkar, Quality measurement and improvement in liver transplantation. J Hepatol, 2018. 68(6): p. 1300-1310.

2. Lee, D.W. and J. Han, Endoscopic management of anastomotic stricture after living-donor liver transplantation. Korean J Intern Med, 2019. 34(2): p. 261-268.

3. Parmar, A., S.M. Vandriel, and V.L. Ng, Health-related quality of life after pediatric liver transplantation: A systematic review. Liver Transpl, 2017. 23(3): p. 361-374. 
4. Yang, L.S., et al., Liver transplantation: a systematic review of long-term quality of life. Liver Int, 2014. 34(9): p. 1298-313.

5. Ling, Q., et al., Association between donor and recipient TCF7L2 gene polymorphisms and the risk of new-onset diabetes mellitus after liver transplantation in a Han Chinese population. J Hepatol, 2013. 58(2): p. 271-7.

6. Bernasconi, E., et al., Airway Microbiota Determines Innate Cell Inflammatory or Tissue Remodeling Profiles in Lung Transplantation. Am J Respir Crit Care Med, 2016. 194(10): p. $1252-1263$.

7. Leithead, J.A. and J.W. Ferguson, Chronic kidney disease after liver transplantation. J Hepatol, 2015. 62(1): p. 243-4.

8. Oldani, G., et al., Chimeric liver transplantation reveals interspecific graft remodelling. J Hepatol, 2018. 69(5): p. 1025-1036.

9. Obara, H., et al., IFN-gamma, produced by NK cells that infiltrate liver allografts early after transplantation, links the innate and adaptive immune responses. Am J Transplant, 2005. 5(9): p. 2094-103.

10. Mossanen, J.C., et al., Chemokine (C-C motif) receptor 2-positive monocytes aggravate the early phase of acetaminophen-induced acute liver injury. Hepatology, 2016. 64(5): p. $1667-1682$.

11. Song, J.L., et al., A new index predicts early allograft dysfunction following living donor liver transplantation: A propensity score analysis. Dig Liver Dis, 2017. 49(11): p. 1225-1232.

12. Kim, N., et al., Combined Detection of Serum IL-10, IL-17, and CXCL10 Predicts Acute Rejection Following Adult Liver Transplantation. Mol Cells, 2016. 39(8): p. 639-44.

13. Sharma, A., et al., Onco-fetal Reprogramming of Endothelial Cells Drives Immunosuppressive Macrophages in Hepatocellular Carcinoma. Cell, 2020. 183(2): p. 377-394 e21.

14. Lotto, J., et al., Single-Cell Transcriptomics Reveals Early Emergence of Liver Parenchymal and Non-parenchymal Cell Lineages. Cell, 2020. 183(3): p. 702-716 e14.

15. Vento-Tormo, R., et al., Single-cell reconstruction of the early maternal-fetal interface in humans. Nature, 2018. 563(7731): p. 347-353.

16. Browaeys, R., W. Saelens, and Y. Saeys, NicheNet: modeling intercellular communication by 
linking ligands to target genes. Nat Methods, 2020. 17(2): p. 159-162.

17. Huang, H., et al., The time-dependent shift in the hepatic graft and recipient macrophage pool following liver transplantation. Cell Mol Immunol, 2020. 17(4): p. 412-414.

18. Zhang, C., et al., A Soluble Form of P Selectin Glycoprotein Ligand 1 Requires Signaling by Nuclear Factor Erythroid 2-Related Factor 2 to Protect Liver Transplant Endothelial Cells Against Ischemia-Reperfusion Injury. Am J Transplant, 2017. 17(6): p. 1462-1475.

19. Ling, Q., et al., The tacrolimus-induced glucose homeostasis imbalance in terms of the liver: From bench to bedside. Am J Transplant, 2020. 20(3): p. 701-713.

20. Zhang, Q., et al., Integrated multiomic analysis reveals comprehensive tumour heterogeneity and novel immunophenotypic classification in hepatocellular carcinomas. Gut, 2019. 68(11): p. 2019-2031.

21. Zhang, M., et al., Single-cell transcriptomic architecture and intercellular crosstalk of human intrahepatic cholangiocarcinoma. J Hepatol, 2020. 73(5): p. 1118-1130.

22. Bonnardel, J., et al., Stellate Cells, Hepatocytes, and Endothelial Cells Imprint the Kupffer Cell Identity on Monocytes Colonizing the Liver Macrophage Niche. Immunity, 2019. 51(4): p. 638-654 e9.

23. Bárcena, C., et al., CD5L is a pleiotropic player in liver fibrosis controlling damage, fibrosis and immune cell content. EBioMedicine, 2019. 43: p. 513-524.

24. $\mathrm{Wu}, \mathrm{H}$., et al., TIM-4 interference in Kupffer cells against CCL4-induced liver fibrosis by mediating Akt1/Mitophagy signalling pathway. Cell Prolif, 2020. 53(1): p. e12731.

25. Wu, X., et al., Soluble CLEC2 Extracellular Domain Improves Glucose and Lipid Homeostasis by Regulating Liver Kupffer Cell Polarization. EBioMedicine, 2015. 2(3): p. 214-24.

26. Yang, X., et al., Overexpressed PLTP in macrophage may promote cholesterol accumulation by prolonged endoplasmic reticulum stress. Med Hypotheses, 2017. 98: p. 45-48.

27. Song, Q., et al., Dissecting intratumoral myeloid cell plasticity by single cell RNA-seq. Cancer Med, 2019. 8(6): p. 3072-3085.

28. Ma, K.L., et al., Inflammatory stress exacerbates lipid accumulation in hepatic cells and fatty livers of apolipoprotein E knockout mice. Hepatology, 2008. 48(3): p. 770-81.

29. Dou, L., et al., Mir-338-3p Mediates Tnf-A-Induced Hepatic Insulin Resistance by Targeting 
PP4r1 to Regulate PP4 Expression. Cell Physiol Biochem, 2017. 41(6): p. 2419-2431.

30. Gilbo, N., et al., Liver graft preconditioning, preservation and reconditioning. Dig Liver Dis, 2016. 48(11): p. 1265-1274.

31. Liu, Y., et al., Activation of YAP attenuates hepatic damage and fibrosis in liver ischemia-reperfusion injury. J Hepatol, 2019. 71(4): p. 719-730.

32. Yazdani, H.O., et al., Exercise training decreases hepatic injury via changes in immune response to liver ischemia/reperfusion in mice. Hepatology, 2020.

33. Patrono, D., et al., Perfusate Analysis During Dual Hypothermic Oxygenated Machine Perfusion of Liver Grafts: Correlations With Donor Factors and Early Outcomes. Transplantation, 2020. 104(9): p. 1929-1942.

34. Xiong, X., et al., Landscape of Intercellular Crosstalk in Healthy and NASH Liver Revealed by Single-Cell Secretome Gene Analysis. Mol Cell, 2019. 75(3): p. 644-660.e5.

35. Robinson, M.W., C. Harmon, and C. O'Farrelly, Liver immunology and its role in inflammation and homeostasis. Cell Mol Immunol, 2016. 13(3): p. 267-76.

36. Chong, A.S., B cells as antigen-presenting cells in transplantation rejection and tolerance. Cell Immunol, 2020. 349: p. 104061.

37. Krenkel, O. and F. Tacke, Liver macrophages in tissue homeostasis and disease. Nat Rev Immunol, 2017. 17(5): p. 306-321.

38. Triantafyllou, E., et al., MerTK expressing hepatic macrophages promote the resolution of inflammation in acute liver failure. Gut, 2018. 67(2): p. 333-347.

39. DeBerge, M., et al., MerTK Cleavage on Resident Cardiac Macrophages Compromises Repair After Myocardial Ischemia Reperfusion Injury. Circ Res, 2017. 121(8): p. 930-940.

40. Kazankov, K., et al., The role of macrophages in nonalcoholic fatty liver disease and nonalcoholic steatohepatitis. Nat Rev Gastroenterol Hepatol, 2018.

41. Sun, H., et al., Accumulation of Tumor-Infiltrating CD49a(+) NK Cells Correlates with Poor Prognosis for Human Hepatocellular Carcinoma. Cancer Immunol Res, 2019. 7(9): p. $1535-1546$.

42. Weng, S.Y., et al., IL-4 Receptor Alpha Signaling through Macrophages Differentially Regulates Liver Fibrosis Progression and Reversal. EBioMedicine, 2018. 29: p. 92-103.

43. Harmon, C., et al., Natural Killer Cells and Liver Transplantation: Orchestrators of Rejection 
or Tolerance? Am J Transplant, 2016. 16(3): p. 751-7.

44. Yeung, O.W., et al., Alternatively activated (M2) macrophages promote tumour growth and invasiveness in hepatocellular carcinoma. J Hepatol, 2015. 62(3): p. 607-16.

45. Ling, Q., et al., New-onset diabetes after liver transplantation: a national report from China Liver Transplant Registry. Liver Int, 2016. 36(5): p. 705-12.

46. Ke, Q.H., et al., New-onset hyperglycemia immediately after liver transplantation: A national survey from China Liver Transplant Registry. Hepatobiliary Pancreat Dis Int, 2018. 17(4): p. $310-315$.

47. MacParland, S.A., et al., Single cell RNA sequencing of human liver reveals distinct intrahepatic macrophage populations. Nat Commun, 2018. 9(1): p. 4383.

48. Han, X., et al., Mapping the Mouse Cell Atlas by Microwell-Seq. Cell, 2018. 172(5): p. 1091-1107.e17.

49. Tosello-Trampont, A.C., et al., Kuppfer cells trigger nonalcoholic steatohepatitis development in diet-induced mouse model through tumor necrosis factor- $\alpha$ production. J Biol Chem, 2012. 287(48): p. 40161-72.

50. Marais, A.D., Apolipoprotein E in lipoprotein metabolism, health and cardiovascular disease. Pathology, 2019. 51(2): p. 165-176.

51. Koyama, Y. and D.A. Brenner, Liver inflammation and fibrosis. J Clin Invest, 2017. 127(1): p. $55-64$. 


\section{FIGURE LEGENDS}

Figure 1 Liver graft recovery in orthotopic liver transplantation mice model. (A) Experimental design in mice. (B) Serum ALT and AST levels in mice. (C) H\&E staining and Suzuki's histological grading of transplanted livers. (D) TUNEL staining of transplanted livers. (E) Neutrophils (LY6G+) and macrophages (F4/80+) infiltration in transplanted livers. (F) The expression of cytokine and endothelial cell activation markers in transplanted livers. ALT, alanine aminotransferase; AST, aspartate aminotransferase; H\&E, hematoxylin and eosin; TUNEL, terminal deoxynucleotidyl transferase-mediated dUTP nick-end labeling.

Figure 2 The cellular hierarchy in the transplanted liver. (A) Schematic diagram of the scRNA-seq analysis workflow. (B) tSNE plots for the cell type identification of single cells (left); Violin plots showing marker genes for 10 distinct cell types (right). (C) Heatmap showing the top 5 DEGs in each cell type. (D) Bar plots showing the proportion of immune cells at pre-LT, 3H, 6H, 12H, 3D, and7D post-LT. scRNA-seq, single-cell RNA sequencing; tSNE, t-distributed stochastic neighbor embedding; DEGs, differentially expressed genes; LT, liver transplantation

Figure 3 The cellular source in the transplanted liver. (A) Schematic diagram of the CyTOF analysis workflow. (B) tSNE plots for the cell type identification of single cells. (C) Heatmap showing the expression levels of markers in distinct clusters. (D) tSNE and pie plots showing the proportion of GDCs and RDCs at pre-LT, 12H, 1D, 3D, 7D and 14D post-LT. (E) Bar plots showing the proportion of GDCs and RDCs in distinct clusters at 14D post-LT. (F) Bar plots showing the proportion of GDCs and RDCs in cluster 1, 23 and 16 at pre-LT, 12H, 1D, 3D, 7D and 14D post-LT. CyTOF, cytometry by time-of-flight; tSNE, t-distributed stochastic neighbor embedding; GDCs, graft-derived cells; RDCs, recipient-derived cells; LT, liver transplantation

Figure 4 Distinct macrophage subclusters in the transplanted liver. (A) tSNE plots of total macrophages (left); Heatmap showing the top 30 DEGs for each subcluster (right). (B) Pathway activity (scored by GSVA) in 5 subclusters. (C) tSNE plots color-coded for the expression (gray to red) of marker genes for the distinct subclusters. The red circle showed subcluster 2. (D) tSNE and bar plots showing the proportion of distinct subclusters at pre-LT, acute phase and stable phase. (E) Volcano plot exhibiting DEGs between subcluster 2 and other macrophages. (F) Scatterplots showing genes upregulated in either acute phase and/or stable phase of subcluster 2 (left); Bar plots showing enriched GO terms of the genes overexpressed by subcluster 2 in the acute phase and stable phase (right). tSNE, t-distributed stochastic neighbor embedding; DEGs, differentially expressed genes; GSVA, gene set variation analysis; LT, liver transplantation; GO, Gene Ontology. 
Figure 5 Macrophage-hepatocyte interplay in the transplanted liver. (A) Heatmap showing the metabolic-related pathway activity (scored by GSVA) in hepatocytes. (B) Circle plot showing links between (1) predicted ligands from subcluster 0 , subcluster 1 , subcluster 2 , subcluster 4 or common, (2) their associated receptors found on hepatocytes and (3) metabolic genes potentially targeted by the ligand-receptor pairs. (C) Potential upstream ligands in subcluster 0 and subcluster 2; (D) Bubble plot showing the expression of potential upstream ligands in subcluster 0 and subcluster 2 at pre-LT, acute phase and stable phase; (E) Cube plot showing the potential receptors expressed by hepatocytes associated with each potential ligands, and their expression at pre-LT, acute phase and stable phase. (F) Network plot showing links between predicted ligands and metabolic genes. GSVA, gene set variation analysis; LT, liver transplantation.

\section{SUPPLEMENTAL}

Figure S1 Function and proportion of each cell type. (A) GO analysis of DEGs in distinct cell types. (B) tSNE plots of distinct cell types at pre-LT, 3H, 6H, 12H, 3D, and7D post-LT. GO, Gene Ontology; DEGs, differentially expressed genes; tSNE, t-distributed stochastic neighbor embedding; LT, liver transplantation 
Table S1 List of primers used.

\begin{tabular}{lll}
\hline Target & Forward primer & Reverse primer \\
\hline Tnfa & 5'-CCCTCACACTCAGATCATCTTCT-3' & 5'-GCTACGACGTGGGCTACAG-3' \\
Il1b & 5'-GCAACTGTTCCTGAACTCAACT-3' & 5'-ATCTTTTGGGGTCCGTCAACT-3' \\
Il10 & 5'-GCTCTTACTGACTGGCATGAG-3' & 5'-CGCAGCTCTAGGAGCATGTG-3' \\
Ifng & 5'-ATGAACGCTACACACTGCATC-3' & 5'-CCATCCTTTTGCCAGTTCCTC-3' \\
E-selectin & 5'-AGTCTAGCGCCTGGATGAAA-3' & 5'-ATCGCCACCAGATGTGTGTA-3' \\
P-selectin & 5'-GTCCACGGAGAGTTTGGTGT-3' & 5'-AAGTGGTGTTCGGACCAAAG-3' \\
Vcam1 & 5'-AAGAGAACCCAGGTGGAGGT-3' & 5'-ACCTAGCGAGGCAAACAAGA-3' \\
Icam1 & 5'-CGAAGGTGGTTCTTCTGAGC-3' & 5'-GTCTGCTGAGACCCCTCTTG-3' \\
Gapdh & 5'-AGGTCGGTGTGAACGGATTTG-3' & 5'-TGTAGACCATGTAGTTGAGGTCA-3' \\
\hline
\end{tabular}


Table S2 Antibody panel

\begin{tabular}{|c|c|c|c|c|c|c|c|c|c|}
\hline Label & Marker & Label & Marker & Label & Marker & Label & Marker & Label & Marker \\
\hline 89Y & CD45 & $147 \mathrm{Sm}$ & Ly6G & $156 \mathrm{Gd}$ & CD1d & $165 \mathrm{Ho}$ & CD161 & $174 \mathrm{Yb}$ & CTLA4 \\
\hline $115 \ln$ & CD3 & $148 \mathrm{Nd}$ & Ly6C & $157 \mathrm{Gd}$ & CD335 & $166 \mathrm{Er}$ & Arginase1(Intra) & $175 \mathrm{Lu}$ & TCRab \\
\hline $139 \mathrm{La}$ & CD44 & $149 \mathrm{Sm}$ & CX3CR1 & $158 \mathrm{Gd}$ & CD19 & $167 \mathrm{Er}$ & CD16 & $176 \mathrm{Yb}$ & MerTK \\
\hline $141 \mathrm{Pr}$ & CD192 & $150 \mathrm{Nd}$ & CD49b & $159 \mathrm{~Tb}$ & $\mathrm{~F} 4 / 80$ & $168 \mathrm{Er}$ & FoxP3(Intra) & $197 \mathrm{Au}$ & CD4 \\
\hline $142 \mathrm{Nd}$ & MHCII & $151 \mathrm{Eu}$ & EGFP(Intra) & $160 \mathrm{Gd}$ & $\mathrm{CD} 274$ & $169 \mathrm{Tm}$ & CD127 & $198 \mathrm{Pt}$ & CD8a \\
\hline $143 \mathrm{Nd}$ & CD366 & $152 \mathrm{Sm}$ & CD11c & 161Dy & iNOS(Intra) & $170 \mathrm{Er}$ & CD49a & 209Bi & CD11b \\
\hline $144 \mathrm{Nd}$ & Tbet(Intra) & $153 \mathrm{Eu}$ & TCRgd & $162 \mathrm{Dy}$ & CD206(Intra) & $171 \mathrm{Yb}$ & GATA3(Intra) & & \\
\hline $145 \mathrm{Nd}$ & GR1 & $154 \mathrm{Sm}$ & CD62L & $163 \mathrm{Dy}$ & $\mathrm{CD} 25$ & $172 \mathrm{Yb}$ & CD279 & & \\
\hline $146 \mathrm{Nd}$ & TER119 & $155 \mathrm{Gd}$ & CD80 & 164Dy & RORgt(Intra) & $173 \mathrm{Yb}$ & CD115 & & \\
\hline
\end{tabular}


Table.S3 Gene sets of interest

\begin{tabular}{|c|c|}
\hline Genes & Sets \\
\hline Acacb & Glucose metabolic process \\
\hline Actn3 & Glucose metabolic process \\
\hline Adipoq & Glucose metabolic process \\
\hline Adipor1 & Glucose metabolic process \\
\hline Akt1 & Glucose metabolic process \\
\hline Akt2 & Glucose metabolic process \\
\hline Arpp19 & Glucose metabolic process \\
\hline $\mathrm{Bad}$ & Glucose metabolic process \\
\hline C1qtnf1 & Glucose metabolic process \\
\hline C1qtnf12 & Glucose metabolic process \\
\hline C1qtnf3 & Glucose metabolic process \\
\hline Clk2 & Glucose metabolic process \\
\hline Cox11 & Glucose metabolic process \\
\hline Cry1 & Glucose metabolic process \\
\hline Ddb1 & Glucose metabolic process \\
\hline Dgat2 & Glucose metabolic process \\
\hline Dgkq & Glucose metabolic process \\
\hline Dusp12 & Glucose metabolic process \\
\hline Dyrk2 & Glucose metabolic process \\
\hline Enpp1 & Glucose metabolic process \\
\hline Ep300 & Glucose metabolic process \\
\hline Epm2aip1 & Glucose metabolic process \\
\hline Erfe & Glucose metabolic process \\
\hline Esrrb & Glucose metabolic process \\
\hline Fam3a & Glucose metabolic process \\
\hline Fam3c & Glucose metabolic process \\
\hline Fbp1 & Glucose metabolic process \\
\hline
\end{tabular}


Foxa2 Glucose metabolic process

Foxk1 Glucose metabolic process

Foxk2 Glucose metabolic process

Foxo1 Glucose metabolic process

Gcg Glucose metabolic process

Gcgr Glucose metabolic process

Gck Glucose metabolic process

Gckr Glucose metabolic process

Gnb3 Glucose metabolic process

Gnmt Glucose metabolic process

Gpld1 Glucose metabolic process

Gpt Glucose metabolic process

Gsk3a Glucose metabolic process

Gsk3b Glucose metabolic process

Igf1 Glucose metabolic process

Igf2 Glucose metabolic process

Igfbp3 Glucose metabolic process

Igfbp4 Glucose metabolic process

Inpp5k Glucose metabolic process

Ins2 Glucose metabolic process

Insr Glucose metabolic process

Irs1 Glucose metabolic process

Irs2 Glucose metabolic process

Kat2a Glucose metabolic process

Kat2b Glucose metabolic process

Khk Glucose metabolic process

Lcmt1 Glucose metabolic process

Lep Glucose metabolic process

Lepr Glucose metabolic process 


\begin{tabular}{|c|c|}
\hline Maea & Glucose metabolic process \\
\hline Midn & Glucose metabolic process \\
\hline Mlycd & Glucose metabolic process \\
\hline Mst1 & Glucose metabolic process \\
\hline Mtor & Glucose metabolic process \\
\hline Ncoa2 & Glucose metabolic process \\
\hline Nfe211 & Glucose metabolic process \\
\hline Nkx1-1 & Glucose metabolic process \\
\hline Nln & Glucose metabolic process \\
\hline Ogt & Glucose metabolic process \\
\hline Pask & Glucose metabolic process \\
\hline Pdk1 & Glucose metabolic process \\
\hline Pdk2 & Glucose metabolic process \\
\hline Pdk3 & Glucose metabolic process \\
\hline Pdk4 & Glucose metabolic process \\
\hline Pfkfb1 & Glucose metabolic process \\
\hline Pfkfb2 & Glucose metabolic process \\
\hline Pgp & Glucose metabolic process \\
\hline Phkg2 & Glucose metabolic process \\
\hline Phlda2 & Glucose metabolic process \\
\hline Pmaip1 & Glucose metabolic process \\
\hline Pomc & Glucose metabolic process \\
\hline Ppara & Glucose metabolic process \\
\hline Ppargc1a & Glucose metabolic process \\
\hline Ppp1ca & Glucose metabolic process \\
\hline Ppp1cb & Glucose metabolic process \\
\hline Ppp1r3a & Glucose metabolic process \\
\hline Ppp1r3b & Glucose metabolic process \\
\hline Ppp1r3c & Glucose metabolic process \\
\hline
\end{tabular}




\begin{tabular}{ll}
\hline Ppp1r3d & Glucose metabolic process \\
Ppp1r3e & Glucose metabolic process \\
Ppp1r3f & Glucose metabolic process \\
Ppp1r3g & Glucose metabolic process \\
Ppp4r3b & Glucose metabolic process \\
Park2 & Glucose metabolic process \\
Pth & Glucose metabolic process \\
Ptpn2 & Glucose metabolic process \\
Ranbp2 & Glucose metabolic process \\
Rgn & Glucose metabolic process \\
Rora & Glucose metabolic process \\
Rorc & Glucose metabolic process \\
Rubcnl & Glucose metabolic process \\
Sdhaf3 & Glucose metabolic process \\
Selenos & Glucose metabolic process
\end{tabular}

Serpina12 Glucose metabolic process

Sesn2 Glucose metabolic process

Sik1 Glucose metabolic process

Sirt1 Glucose metabolic process

Sirt7 Glucose metabolic process

Slc25a12 Glucose metabolic process

Slc35b4 Glucose metabolic process

Slc45a3 Glucose metabolic process

Soga1 Glucose metabolic process

Sorbs1 Glucose metabolic process

Src Glucose metabolic process

Tff3 Glucose metabolic process

Tigar Glucose metabolic process

Trp53 Glucose metabolic process 
Usp7 Glucose metabolic process

Wdr5 Glucose metabolic process

Zmpste24 Glucose metabolic process

Acaca Insulin signaling pathway

Akt3 Insulin signaling pathway

Araf Insulin signaling pathway

Braf Insulin signaling pathway

Calm1 Insulin signaling pathway

Calm2 Insulin signaling pathway

Calml3 Insulin signaling pathway

Calm4 Insulin signaling pathway

Cbl Insulin signaling pathway

Cblb Insulin signaling pathway

Cblc Insulin signaling pathway

Crk Insulin signaling pathway

Crkl Insulin signaling pathway

Eif4e Insulin signaling pathway

Eif4e1b Insulin signaling pathway

Eif4e2 Insulin signaling pathway

Eif4ebp1 Insulin signaling pathway

Elk1 Insulin signaling pathway

Exoc7 Insulin signaling pathway

Fasn Insulin signaling pathway

Fbp2 Insulin signaling pathway

Flot1 Insulin signaling pathway

Flot2 Insulin signaling pathway

G6pc Insulin signaling pathway

G6pc2 Insulin signaling pathway

Grb2 Insulin signaling pathway 


\begin{tabular}{|c|c|}
\hline Gys1 & Insulin signaling pathway \\
\hline Gys2 & Insulin signaling pathway \\
\hline Hk1 & Insulin signaling pathway \\
\hline Hk2 & Insulin signaling pathway \\
\hline Hk3 & Insulin signaling pathway \\
\hline Hras & Insulin signaling pathway \\
\hline Ikbkb & Insulin signaling pathway \\
\hline Inpp5d & Insulin signaling pathway \\
\hline Irs4 & Insulin signaling pathway \\
\hline Kras & Insulin signaling pathway \\
\hline Lipe & Insulin signaling pathway \\
\hline Map2k1 & Insulin signaling pathway \\
\hline Map2k2 & Insulin signaling pathway \\
\hline Mapk1 & Insulin signaling pathway \\
\hline Mapk10 & Insulin signaling pathway \\
\hline Mapk3 & Insulin signaling pathway \\
\hline Mapk8 & Insulin signaling pathway \\
\hline Mapk9 & Insulin signaling pathway \\
\hline Mknk1 & Insulin signaling pathway \\
\hline Mknk2 & Insulin signaling pathway \\
\hline Nras & Insulin signaling pathway \\
\hline Pck1 & Insulin signaling pathway \\
\hline Pck2 & Insulin signaling pathway \\
\hline Pde3a & Insulin signaling pathway \\
\hline Pde3b & Insulin signaling pathway \\
\hline Pdpk1 & Insulin signaling pathway \\
\hline Phka1 & Insulin signaling pathway \\
\hline Phka2 & Insulin signaling pathway \\
\hline Phkb & Insulin signaling pathway \\
\hline
\end{tabular}




\begin{tabular}{|c|c|}
\hline Phkg1 & Insulin signaling pathway \\
\hline Pik3ca & Insulin signaling pathway \\
\hline Pik3cb & Insulin signaling pathway \\
\hline Pik3cd & Insulin signaling pathway \\
\hline Pik3cg & Insulin signaling pathway \\
\hline Pik3r1 & Insulin signaling pathway \\
\hline Pik3r2 & Insulin signaling pathway \\
\hline Pik3r3 & Insulin signaling pathway \\
\hline Pik3r5 & Insulin signaling pathway \\
\hline Pklr & Insulin signaling pathway \\
\hline Gm5601 & Insulin signaling pathway \\
\hline Prkaa1 & Insulin signaling pathway \\
\hline Prkaa2 & Insulin signaling pathway \\
\hline Prkab1 & Insulin signaling pathway \\
\hline Prkab2 & Insulin signaling pathway \\
\hline Prkaca & Insulin signaling pathway \\
\hline Prkacb & Insulin signaling pathway \\
\hline Prkag1 & Insulin signaling pathway \\
\hline Prkag2 & Insulin signaling pathway \\
\hline Prkag3 & Insulin signaling pathway \\
\hline Prkar1a & Insulin signaling pathway \\
\hline Prkarlb & Insulin signaling pathway \\
\hline Prkar2a & Insulin signaling pathway \\
\hline Prkar2b & Insulin signaling pathway \\
\hline Prkci & Insulin signaling pathway \\
\hline Prkcz & Insulin signaling pathway \\
\hline Prkx & Insulin signaling pathway \\
\hline Ptpn1 & Insulin signaling pathway \\
\hline Ptprf & Insulin signaling pathway \\
\hline
\end{tabular}


Pygb Insulin signaling pathway

Pygl Insulin signaling pathway

Pygm Insulin signaling pathway

Raf1 Insulin signaling pathway

Rapgef1 Insulin signaling pathway

Rheb Insulin signaling pathway

Rhoq Insulin signaling pathway

Rps6 Insulin signaling pathway

Rps6kb1 Insulin signaling pathway

Rps6kb2 Insulin signaling pathway

Rptor Insulin signaling pathway

Sh2b2 Insulin signaling pathway

Shc1 Insulin signaling pathway

Shc2 Insulin signaling pathway

Shc3 Insulin signaling pathway

Shc4 Insulin signaling pathway

Slc2a4 Insulin signaling pathway

Socs1 Insulin signaling pathway

Socs2 Insulin signaling pathway

Socs3 Insulin signaling pathway

Socs4 Insulin signaling pathway

Sos1 Insulin signaling pathway

Sos2 Insulin signaling pathway

Srebf1 Insulin signaling pathway

Trip10 Insulin signaling pathway

Tsc1 Insulin signaling pathway

Tsc2 Insulin signaling pathway

Abca1 Lipid homeostasis

Abca12 Lipid homeostasis 


\begin{tabular}{|c|c|}
\hline Abca2 & Lipid homeostasis \\
\hline Abcb11 & Lipid homeostasis \\
\hline Abcb4 & Lipid homeostasis \\
\hline Abcd1 & Lipid homeostasis \\
\hline Abcg1 & Lipid homeostasis \\
\hline Abcg5 & Lipid homeostasis \\
\hline Abcg8 & Lipid homeostasis \\
\hline Abhd4 & Lipid homeostasis \\
\hline Abhd5 & Lipid homeostasis \\
\hline Abhd8 & Lipid homeostasis \\
\hline Acox1 & Lipid homeostasis \\
\hline Acox 2 & Lipid homeostasis \\
\hline Acox3 & Lipid homeostasis \\
\hline Acoxl & Lipid homeostasis \\
\hline Acsm1 & Lipid homeostasis \\
\hline Acsm3 & Lipid homeostasis \\
\hline Adck1 & Lipid homeostasis \\
\hline Adora1 & Lipid homeostasis \\
\hline Akr1c21 & Lipid homeostasis \\
\hline Angpt13 & Lipid homeostasis \\
\hline Angpt14 & Lipid homeostasis \\
\hline Angpt18 & Lipid homeostasis \\
\hline Apoa1 & Lipid homeostasis \\
\hline Apoa2 & Lipid homeostasis \\
\hline Apoa4 & Lipid homeostasis \\
\hline Apoa5 & Lipid homeostasis \\
\hline Apob & Lipid homeostasis \\
\hline Apoc2 & Lipid homeostasis \\
\hline Apoc3 & Lipid homeostasis \\
\hline
\end{tabular}




\begin{tabular}{ll}
\hline Apoc4 & Lipid homeostasis \\
Apoe & Lipid homeostasis \\
Apom & Lipid homeostasis \\
Asgr2 & Lipid homeostasis \\
Atp13a2 & Lipid homeostasis \\
Cav1 & Lipid homeostasis \\
Cav3 & Lipid homeostasis \\
Cd24a & Lipid homeostasis \\
Cebpa & Lipid homeostasis \\
Ces1d & Lipid homeostasis \\
Commd9 & Lipid homeostasis \\
Cyp39a1 & Lipid homeostasis \\
Cyp7a1 & Lipid homeostasis \\
Cyp7b1 & Lipid homeostasis \\
Ditm2 & Lipid homeostasis \\
Fip & Lipid homeostasis \\
Dgat1 & Lipid homeostasis \\
Disp3 & Lipid homeostasis \\
Ehd1 & Lipid homeostasis \\
Fnpp7 & Lipid homeostasis \\
Ephx2 & Lipid homeostasis \\
Fabp3 & Lipid homeostasis \\
Lipid
\end{tabular}




\begin{tabular}{ll}
\hline Gramd1b & Lipid homeostasis \\
Hdac9 & Lipid homeostasis \\
Hnf4a & Lipid homeostasis \\
Il18 & Lipid homeostasis \\
Insig1 & Lipid homeostasis \\
Lamtor1 & Lipid homeostasis \\
Lcat & Lipid homeostasis \\
Ldlr & Lipid homeostasis \\
Ldlrap1 & Lipid homeostasis \\
Lima1 & Lipid homeostasis \\
Lipc & Lipid homeostasis \\
Lipg & Lipid homeostasis \\
Lr1i2 & Lipid homeostasis \\
Nr1h3 & Lipid homeostasis \\
Lrp5 & Lipid homeostasis \\
Mall & Lipid homeostasis \\
Malrd1 & Lipid homeostasis \\
Med13 & Lipid homeostasis \\
Mlxipl & Lipid homeostasis \\
Mttp & Lipid homeostasis \\
Mylip & Lipid homeostasis \\
Npc1 & Lipid homeostasis \\
Npc & Lipid homeostasis \\
Lipid homeostasis \\
Lipid homeostasis \\
Lipid homeostasis \\
Nr1d
\end{tabular}




\begin{tabular}{ll}
\hline Nr5a2 & Lipid homeostasis \\
Nus1 & Lipid homeostasis \\
Ormd11 & Lipid homeostasis \\
Ormd12 & Lipid homeostasis \\
Ormd13 & Lipid homeostasis \\
Pcsk9 & Lipid homeostasis \\
Pla2g10 & Lipid homeostasis \\
Pla2g12b & Lipid homeostasis \\
Plscr3 & Lipid homeostasis \\
Pnpla1 & Lipid homeostasis \\
Pnpla2 & Lipid homeostasis \\
Pnpla3 & Lipid homeostasis \\
Pnpla5 & Lipid homeostasis \\
Pnpla8 & Lipid homeostasis \\
Pold1 & Lipid homeostasis \\
Pparg & Lipid homeostasis \\
Raly & Lipid homeostasis \\
Rbp1 & Lipid homeostasis \\
Rcn3 & Lipid homeostasis \\
Rtn4 & Lipid homeostasis \\
Scarb1 & Lipid homeostasis \\
Sec24a & Lipid homeostasis \\
Soat1 & Lipid homeostasis \\
Lipid homeostasis \\
Lipid homeostasis \\
Lipid homeostasis \\
Lipid homeostasis \\
Tlcd
\end{tabular}


bioRxiv preprint doi: https://doi.org/10.1101/2021.01.06.425562; this version posted January 9, 2021. The copyright holder for this preprint (which was not certified by peer review) is the author/funder. All rights reserved. No reuse allowed without permission.

\begin{tabular}{ll}
\hline Tm6sf2 & Lipid homeostasis \\
Tmem97 & Lipid homeostasis \\
Ttc39b & Lipid homeostasis \\
Usf1 & Lipid homeostasis \\
Usf2 & Lipid homeostasis \\
Vdr & Lipid homeostasis \\
Xbp1 & Lipid homeostasis \\
Zbtb20 & Lipid homeostasis
\end{tabular}


A
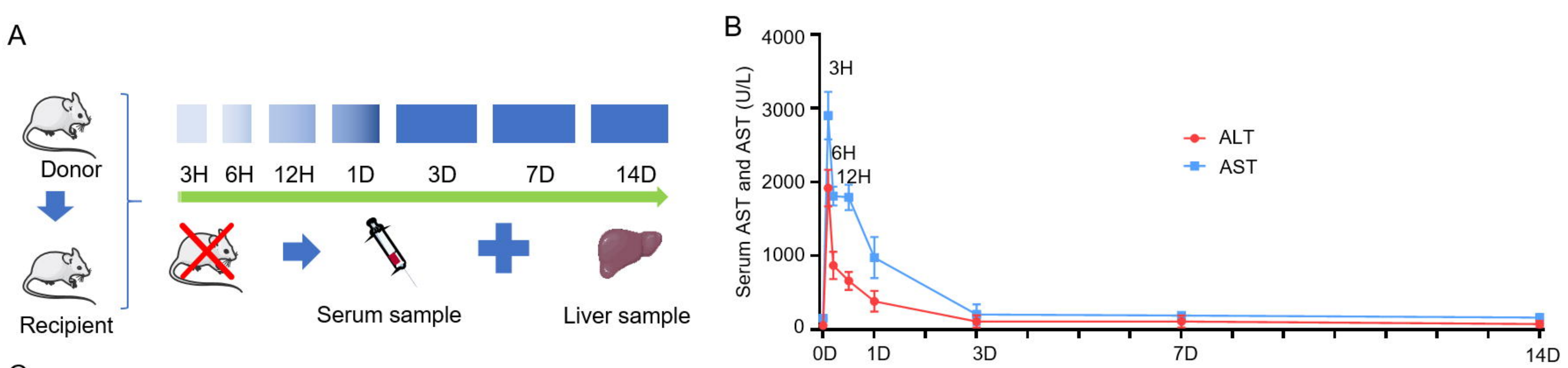

C
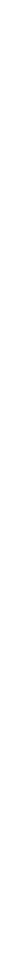

D

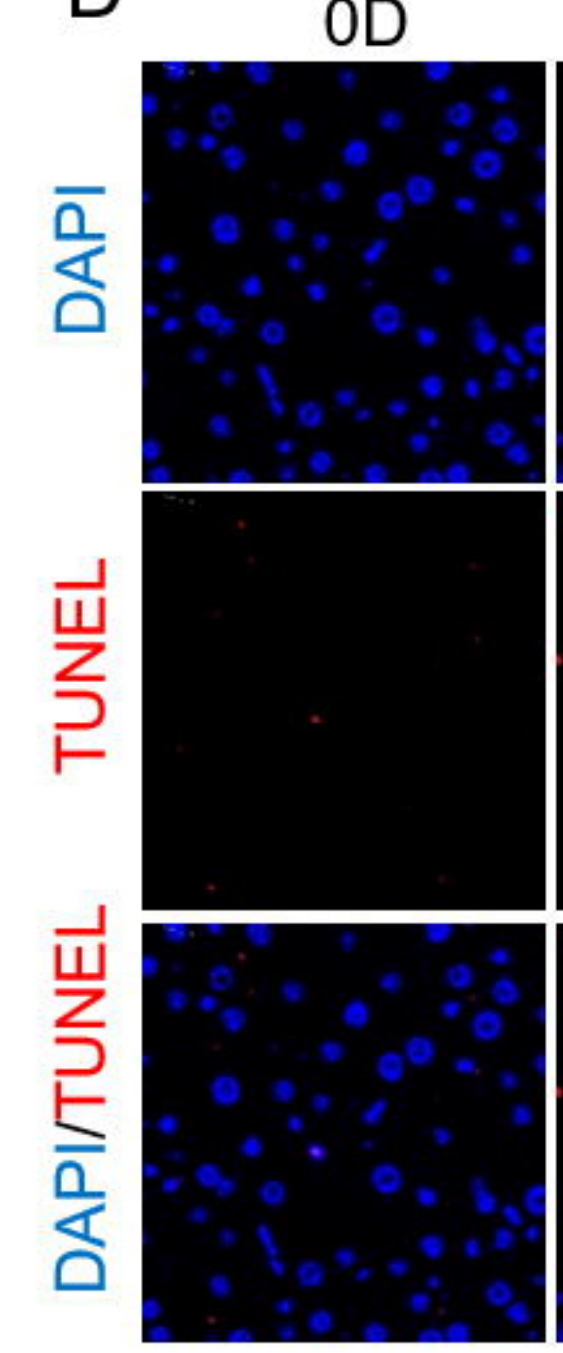

$3 \mathrm{H}$

$6 \mathrm{H}$

$12 \mathrm{H}$

1D
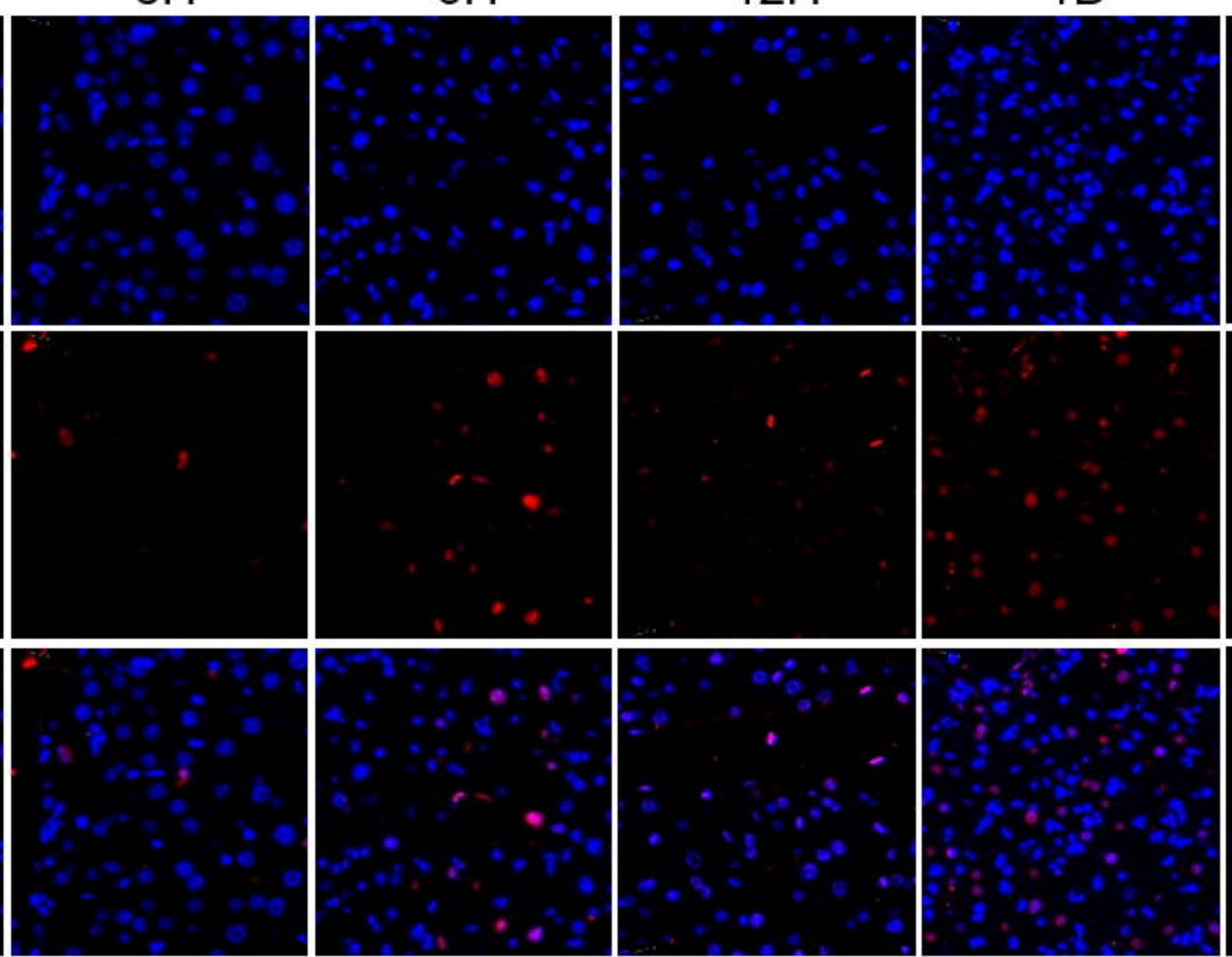

$6 \mathrm{H}$
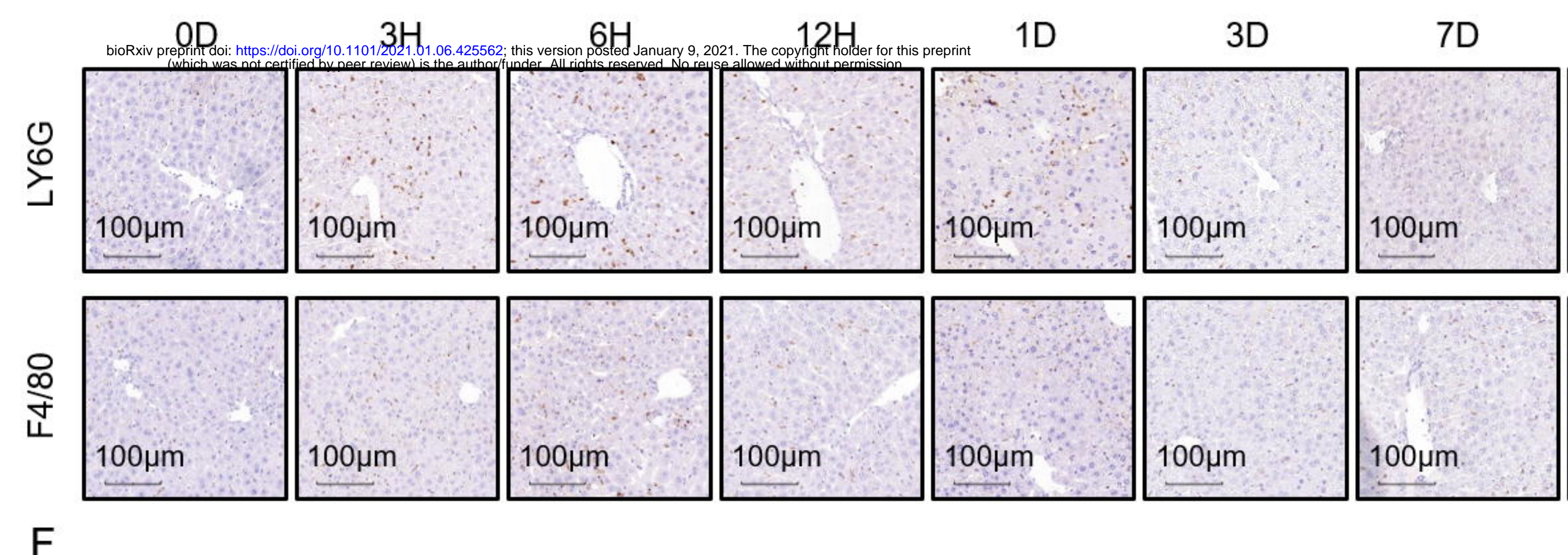

$\underset{\square}{\infty}$
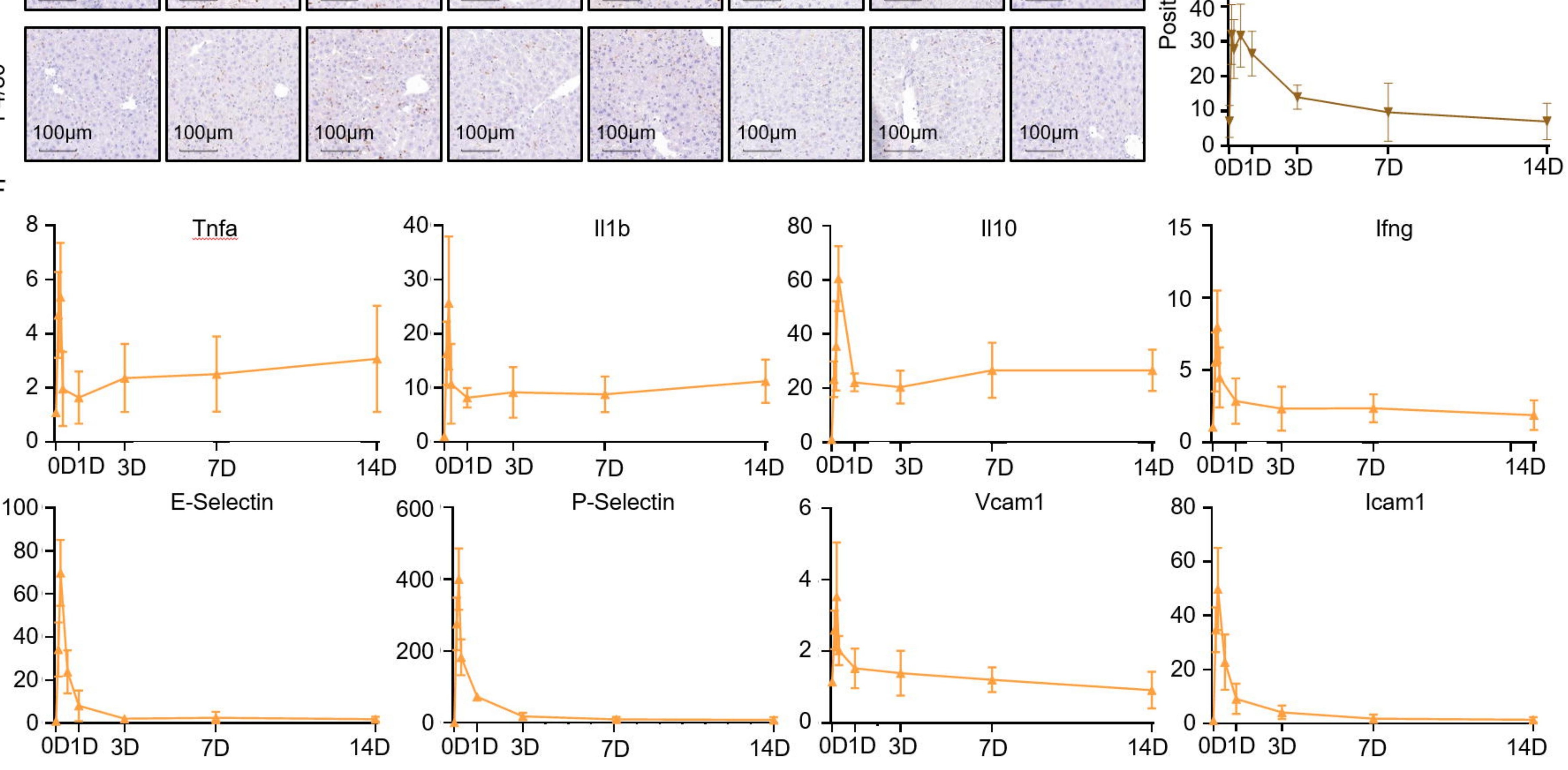
A

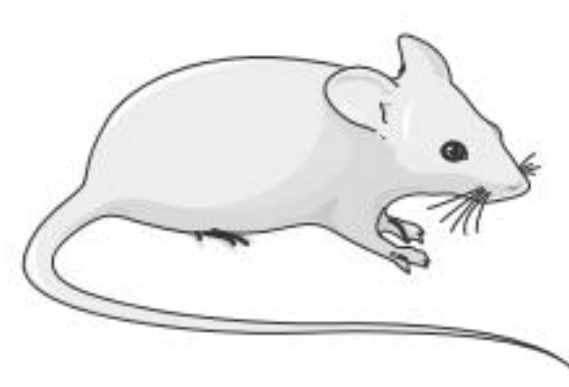

LT model

B

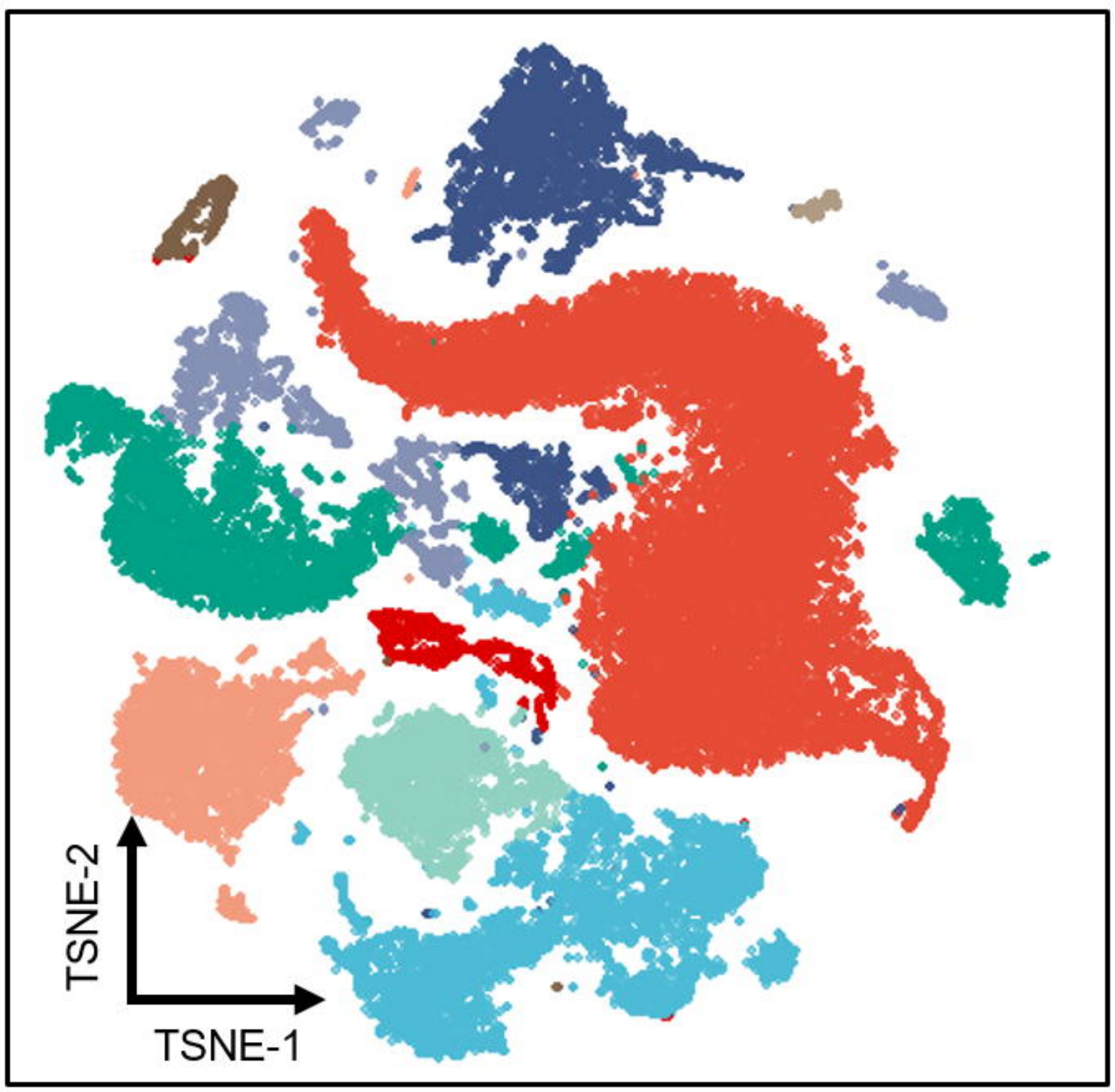

Cluster

- Granulocyte

T cell

Macrophage

- Endothelial cell

B cell

DC

NK

- Hepatocyte

Biliary epithelia cell

Fibroblast

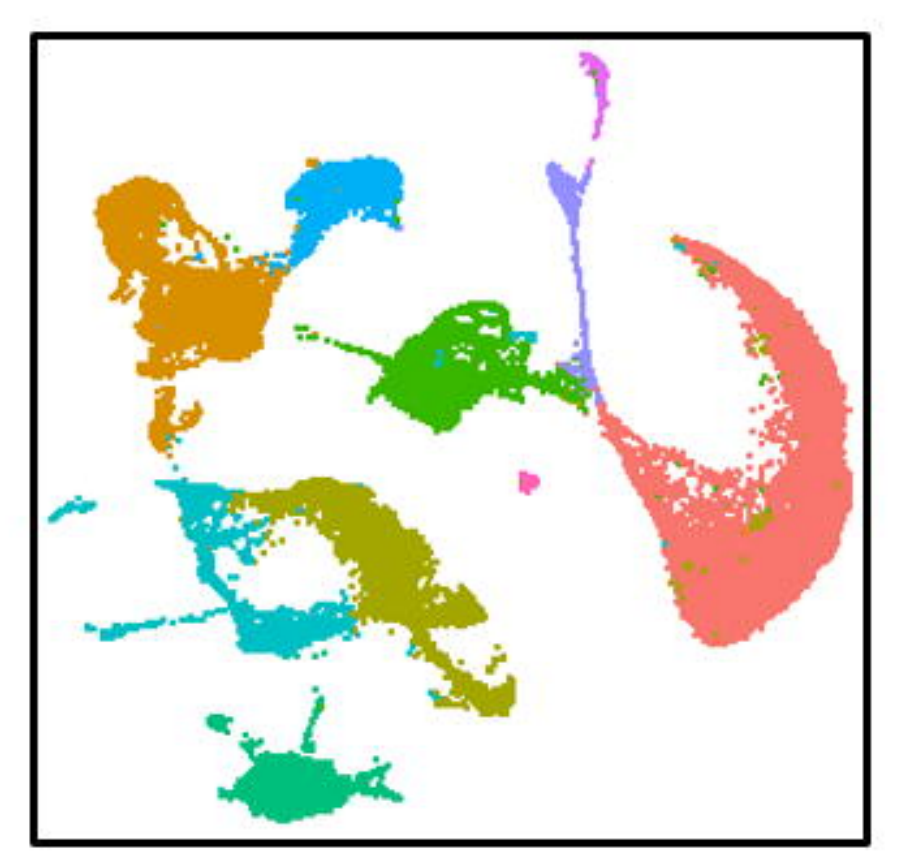

Cell atlas

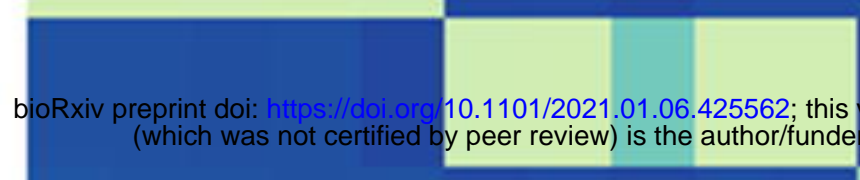

D

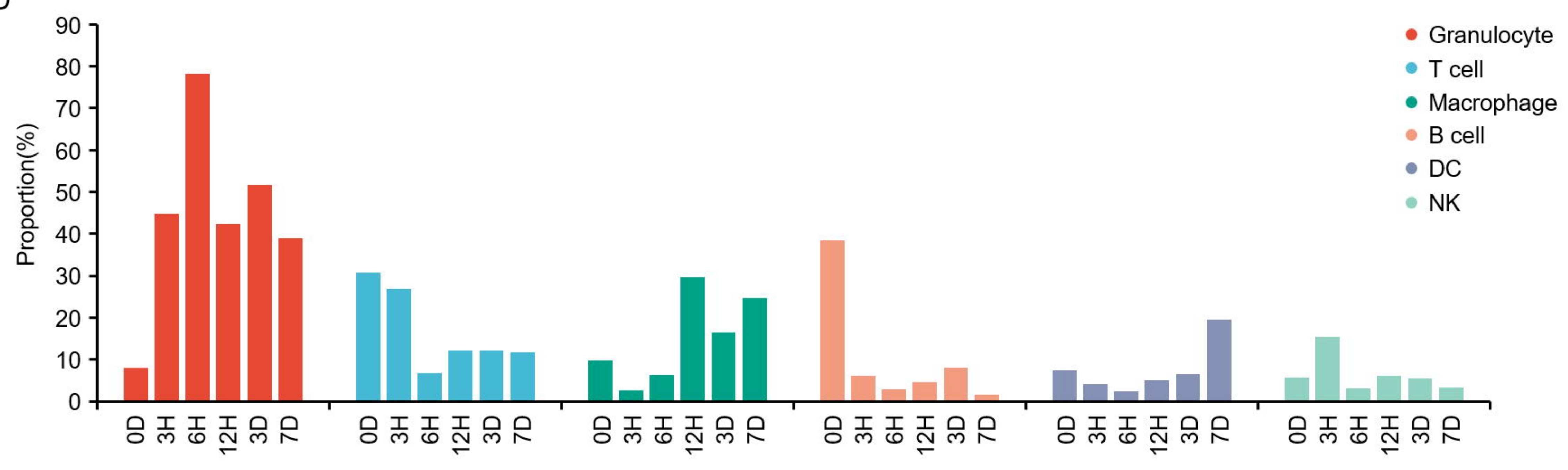


A

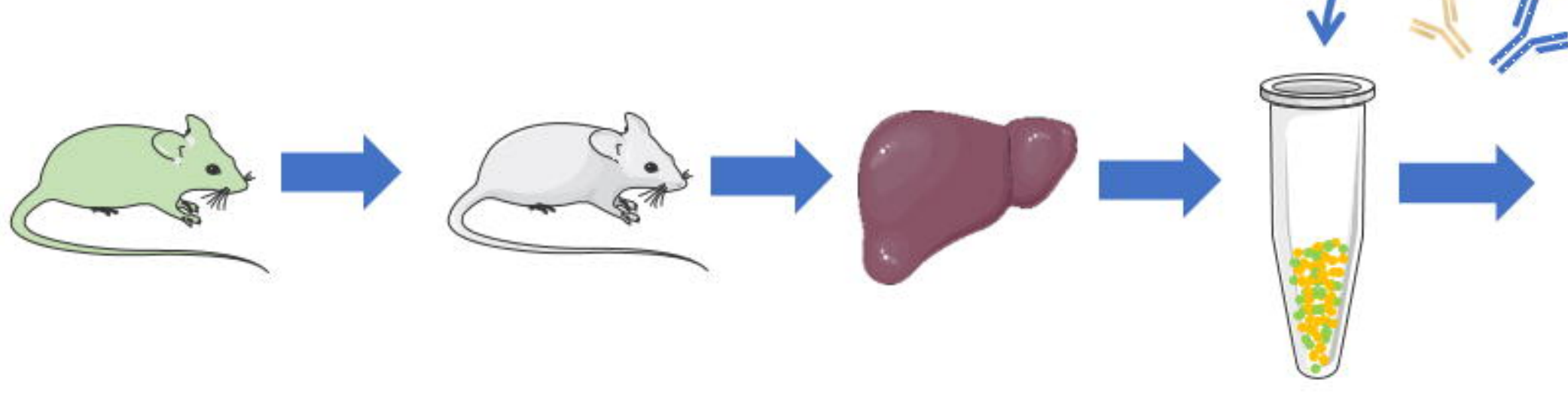

eGFP $^{+}$mice
WT mice

Digestion antibody staining

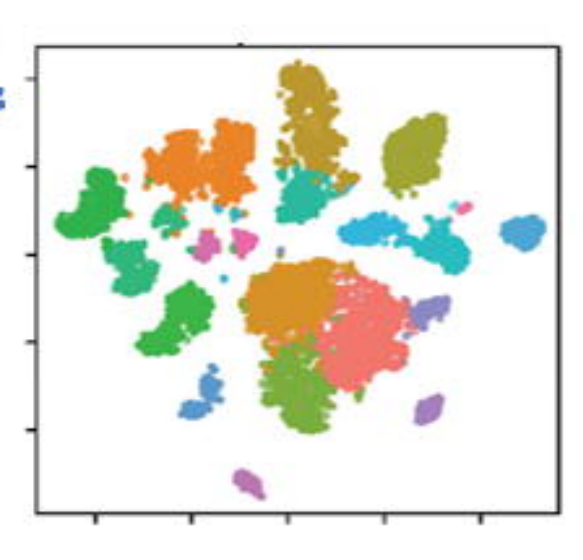

CyTOF analysis
B

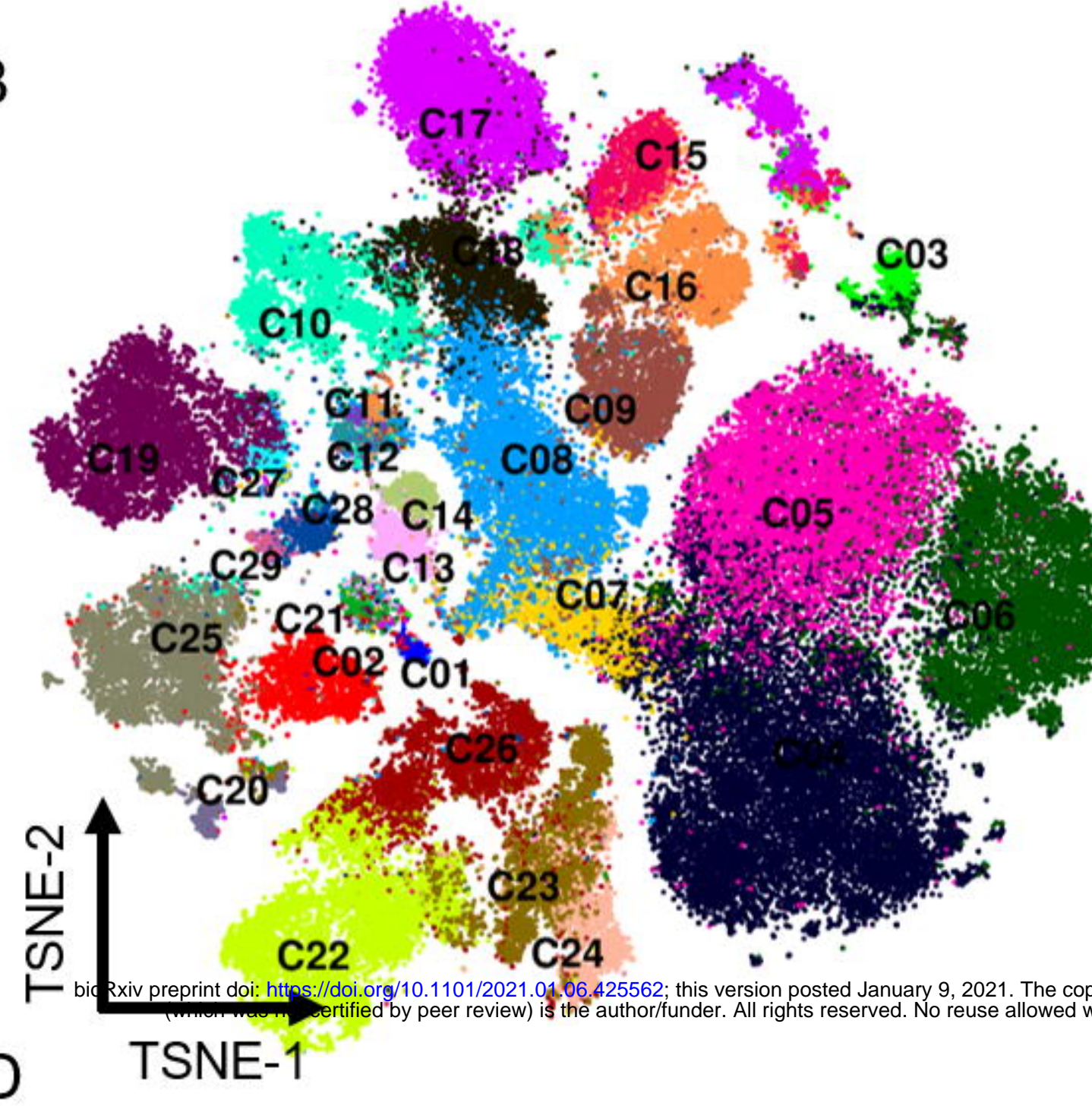

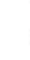

(19) B cells

(27) $B$ cells $\mathrm{CD}^{+} \mathrm{T}$ cells

(23) $\mathrm{CD}^{+} \mathrm{T}$ cells

(24) $\mathrm{CD}^{+} \mathrm{T}$ cells

(26) $\mathrm{CD}^{+} \mathrm{T}$ cells

(20) $\mathrm{y}$ T cells

(10) DC

(11) DC

(12) DC

(28) DC

(29) DC

(1) NK

$2 \mathrm{NK}$

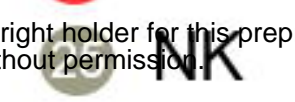
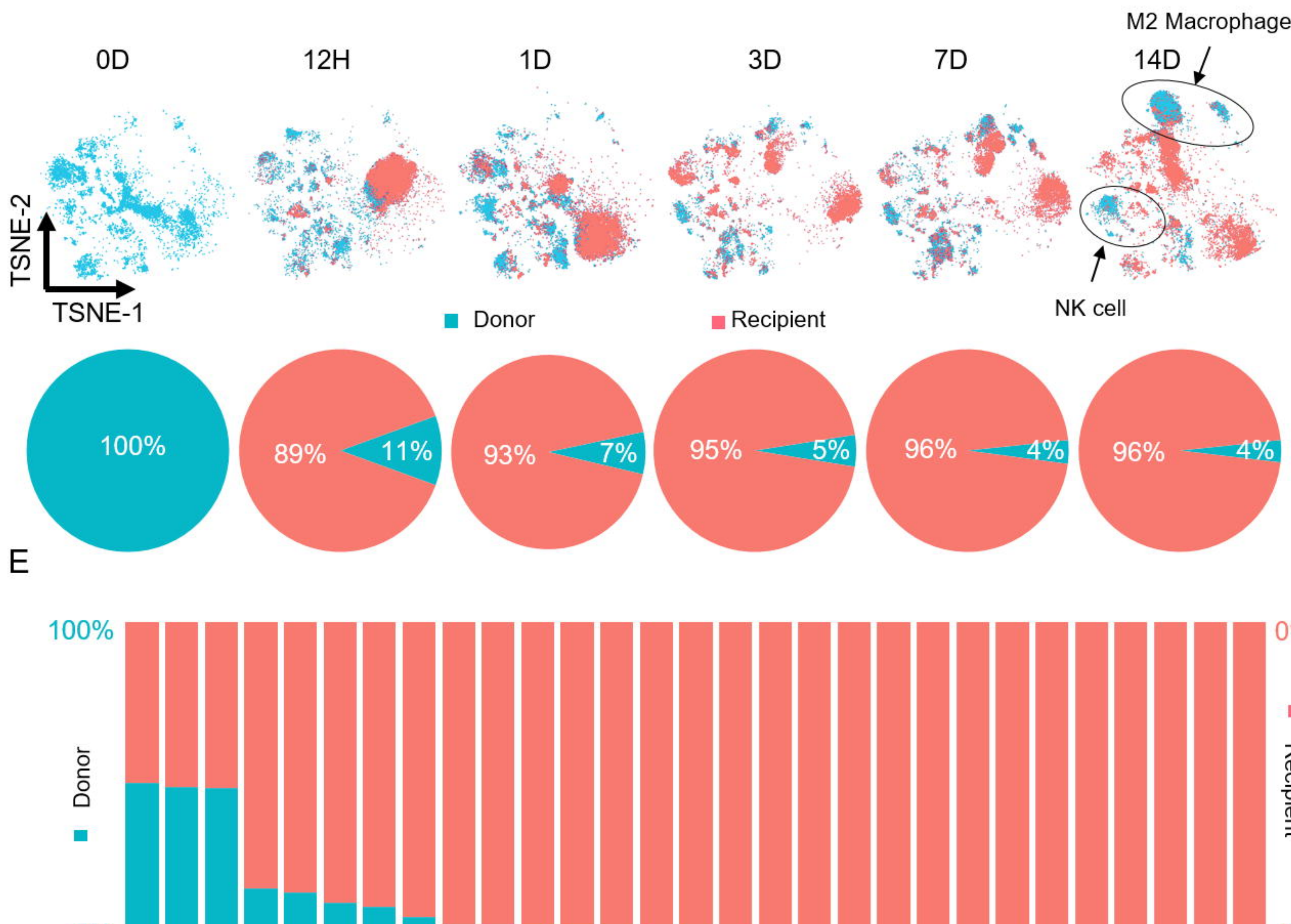

$0 \%$

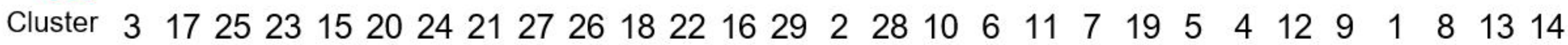

(13) M0 macrophage

(14) M0 macrophage

(9) M1 macrophage

(15) M1 macrophage

(16) M1 macrophage

(17) M2 macrophage

18 M2 macrophage

(3) M2 macrophage

(5) Granulocytes

(6) Granulocytes

(4) Monocytes

(7) Monocytes

8 Monocytes

(21) Other

C $\begin{array}{lllllllllllllll}1 & 3 & 5 & 7 & 9 & 11 & 13 & 15 & 17 & 20 & 24 & 26 & 27 & 29 & 25\end{array}$

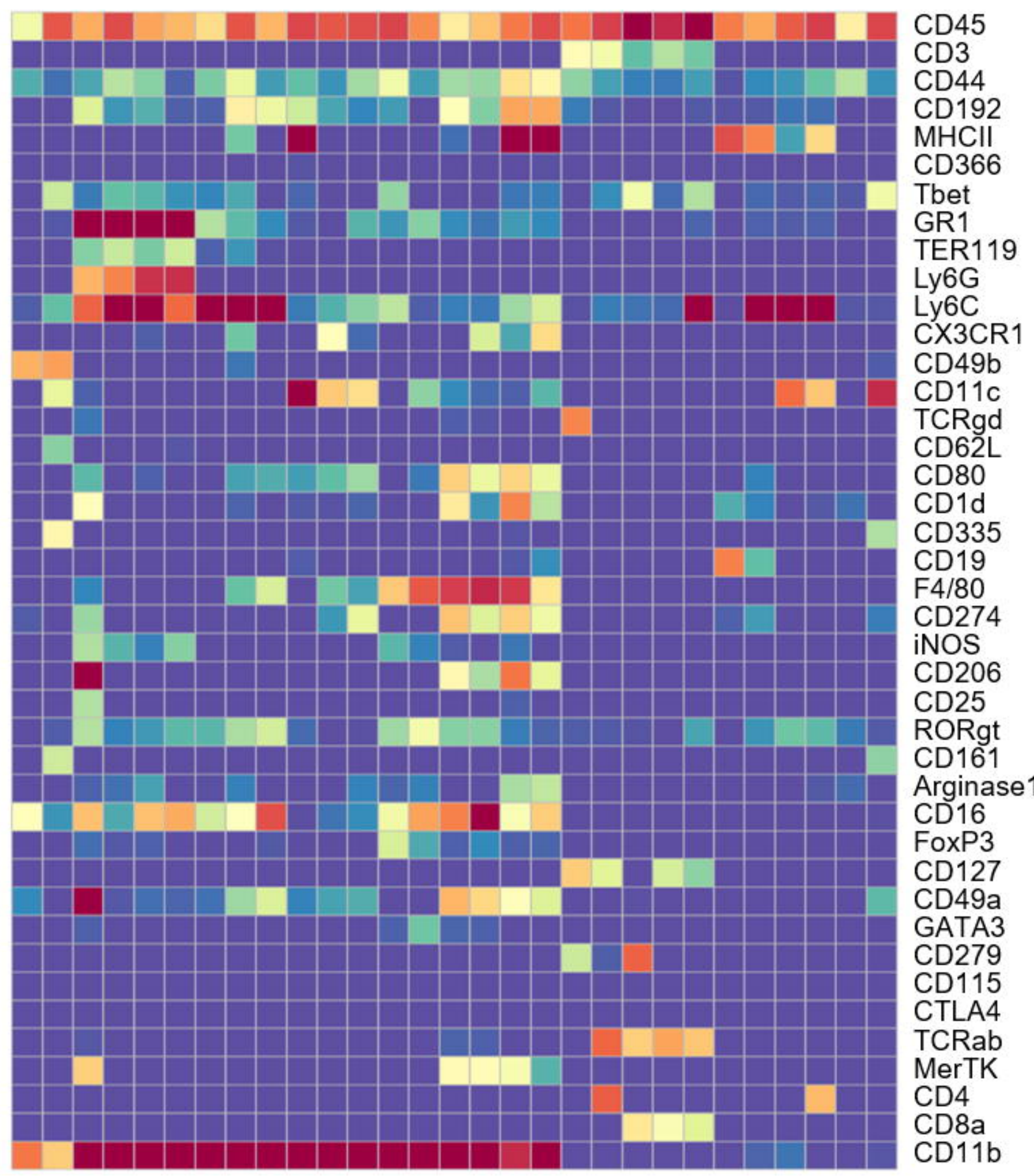

$\begin{array}{lllllllllllllll}\text { Cluster } & 2 & 4 & 6 & 8 & 10 & 12 & 14 & 16 & 18 & 22 & 23 & 19 & 28 & 21\end{array}$

$\mathrm{F}$

Cluster 3

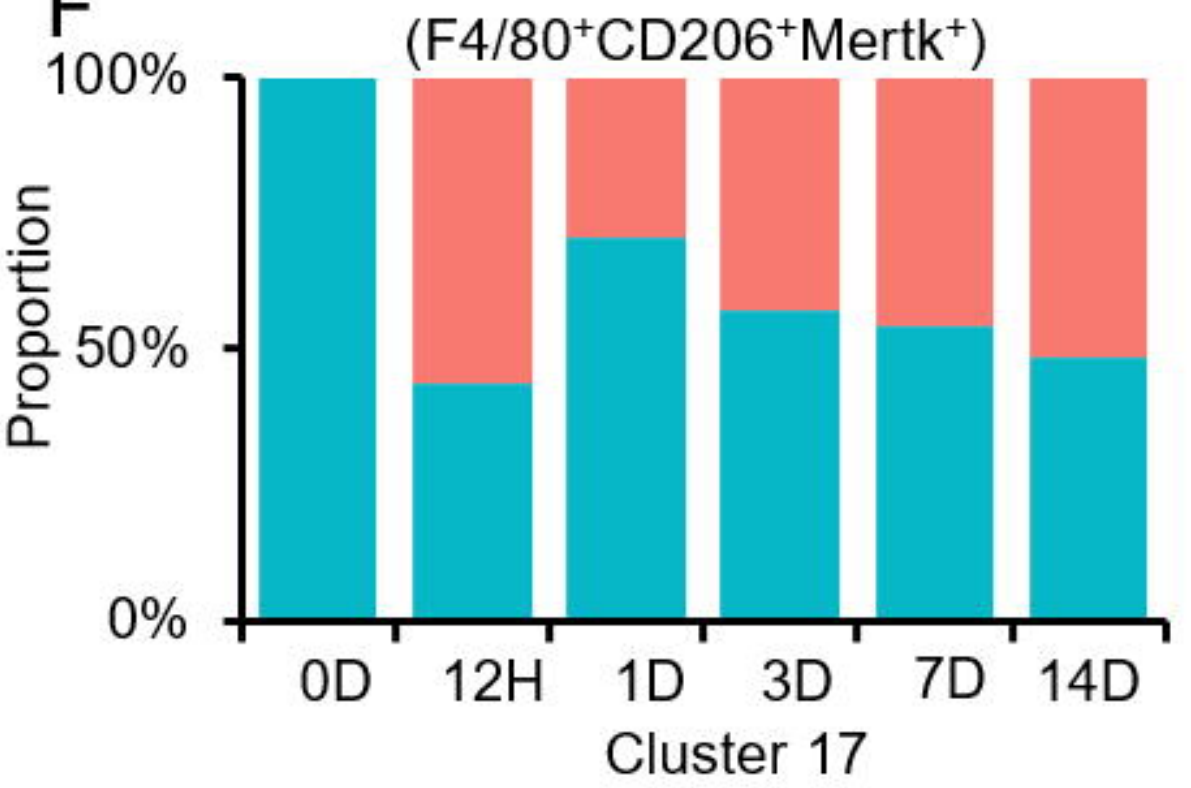

$\left(\mathrm{F} 4 / 80^{+} \mathrm{CD} 206^{+}\right.$Mertk $\left.^{+}\right)$

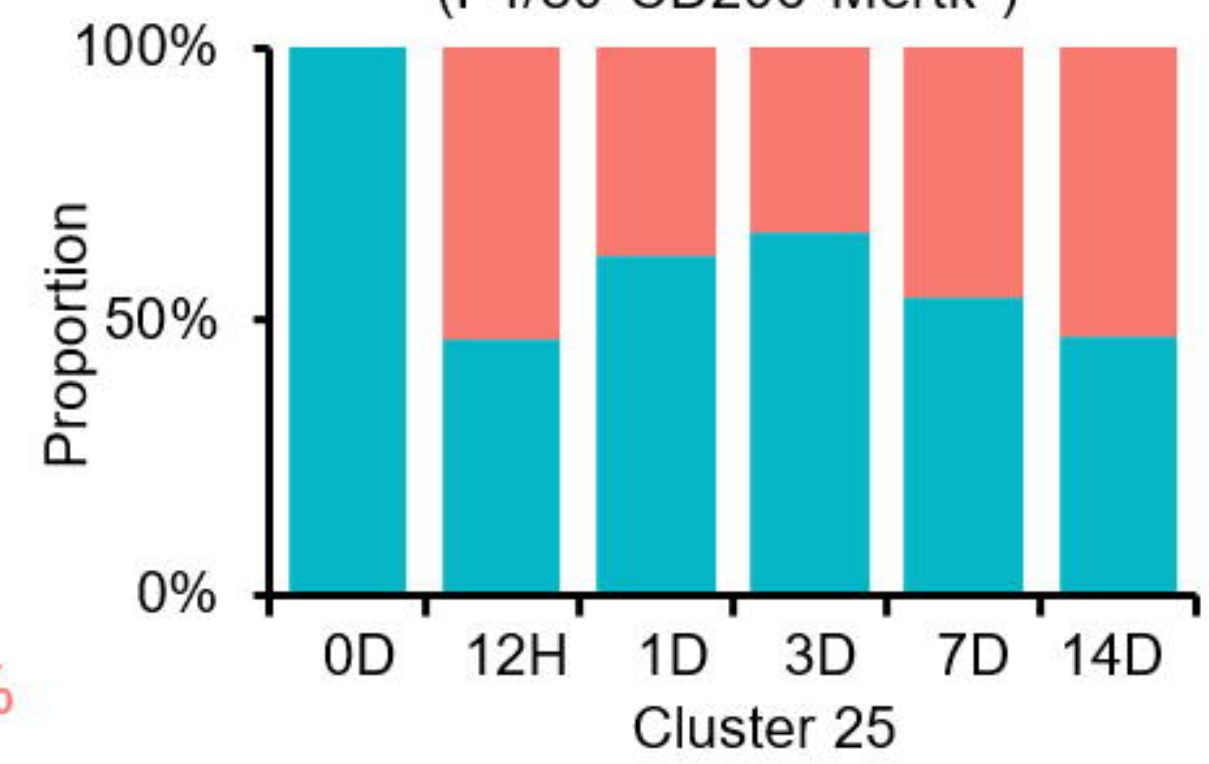
$\left(\mathrm{CD} 161^{+} \mathrm{CD}^{-2} \mathrm{a}^{+}{ }^{\mathrm{C}} \mathrm{CD} 49 \mathrm{~b}-\right)$

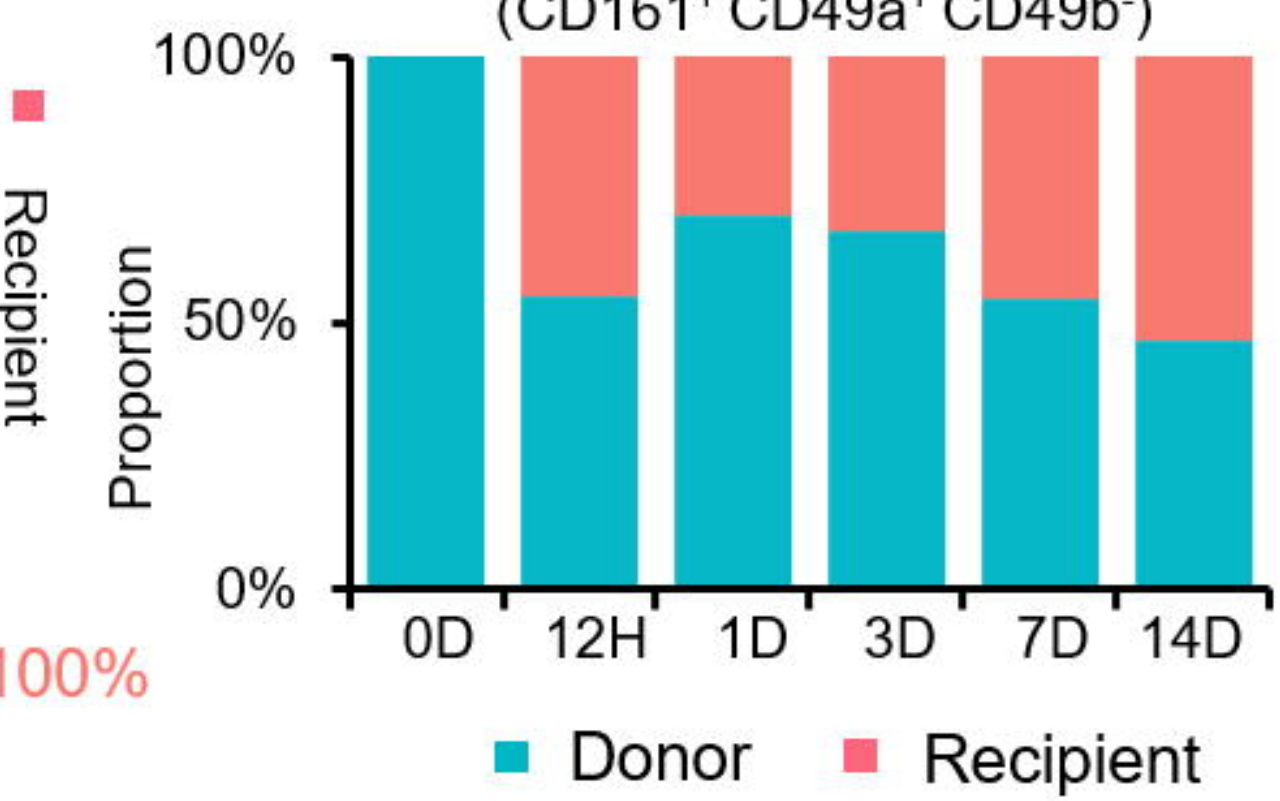


A

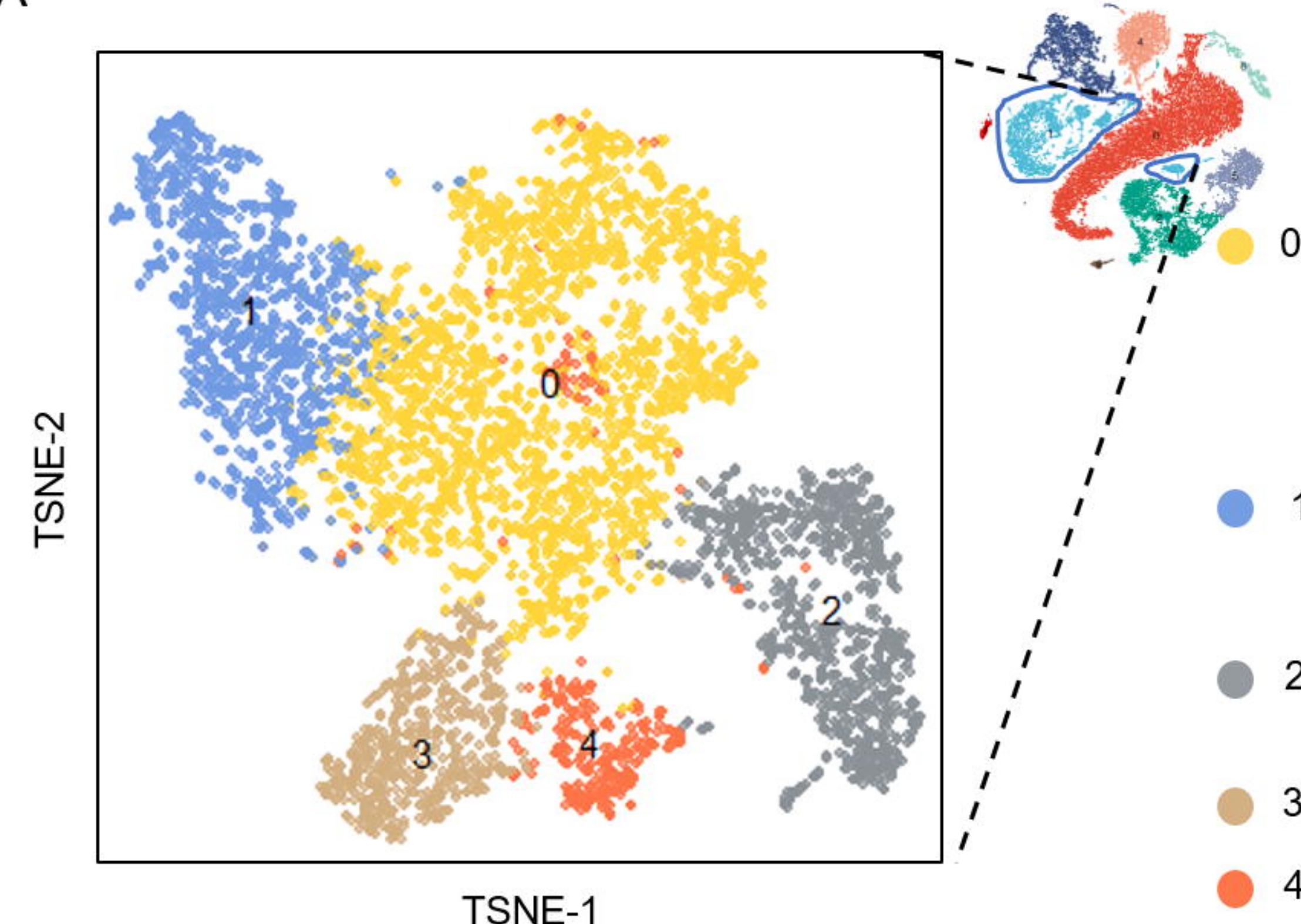

B

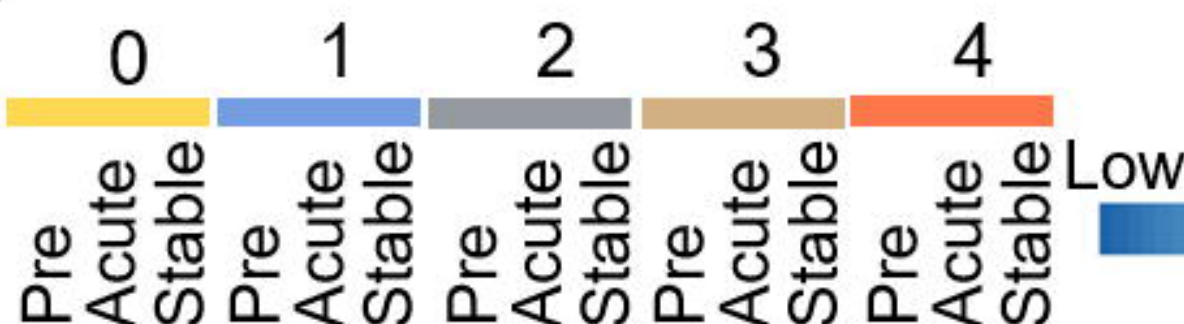

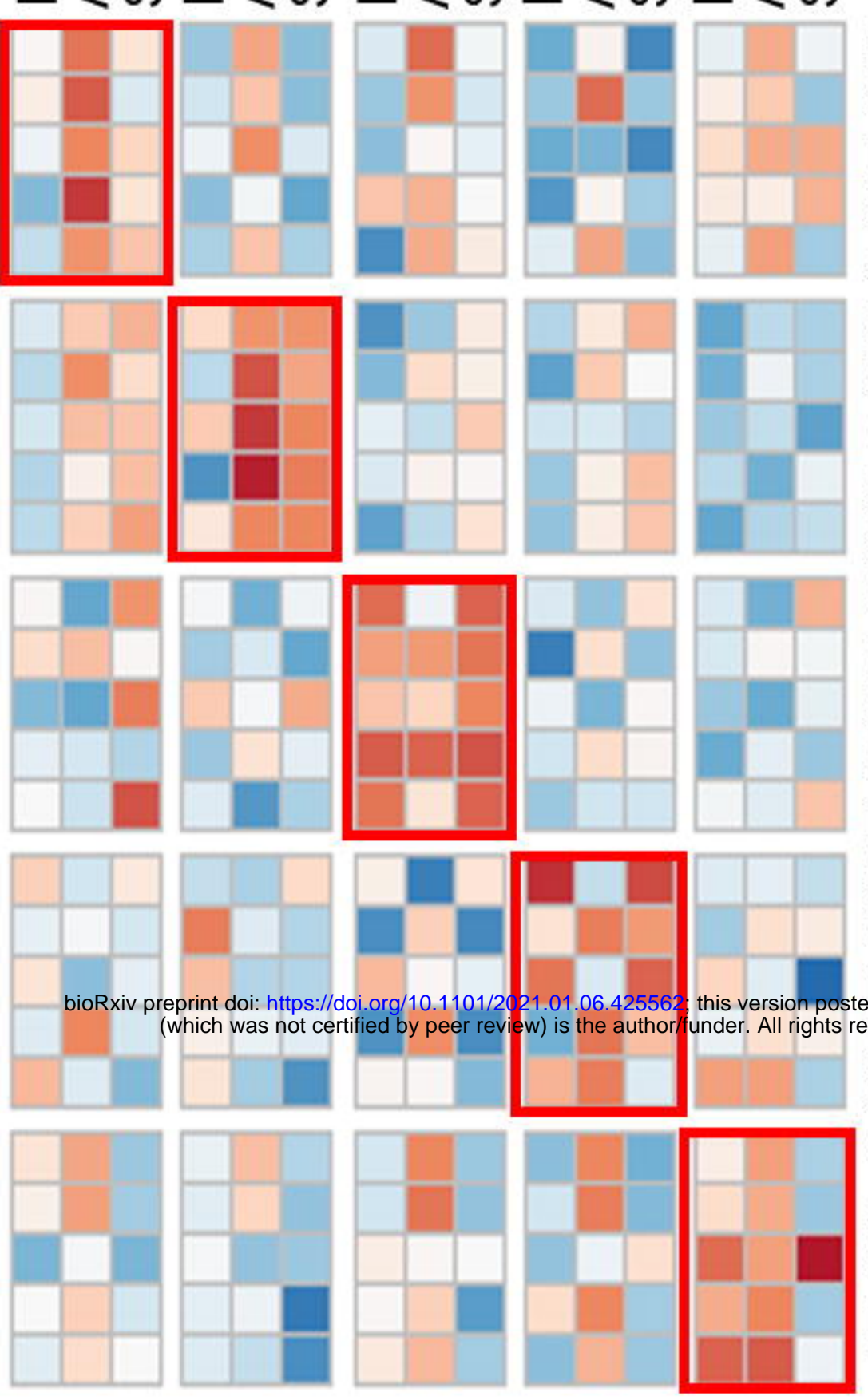

D

Pre

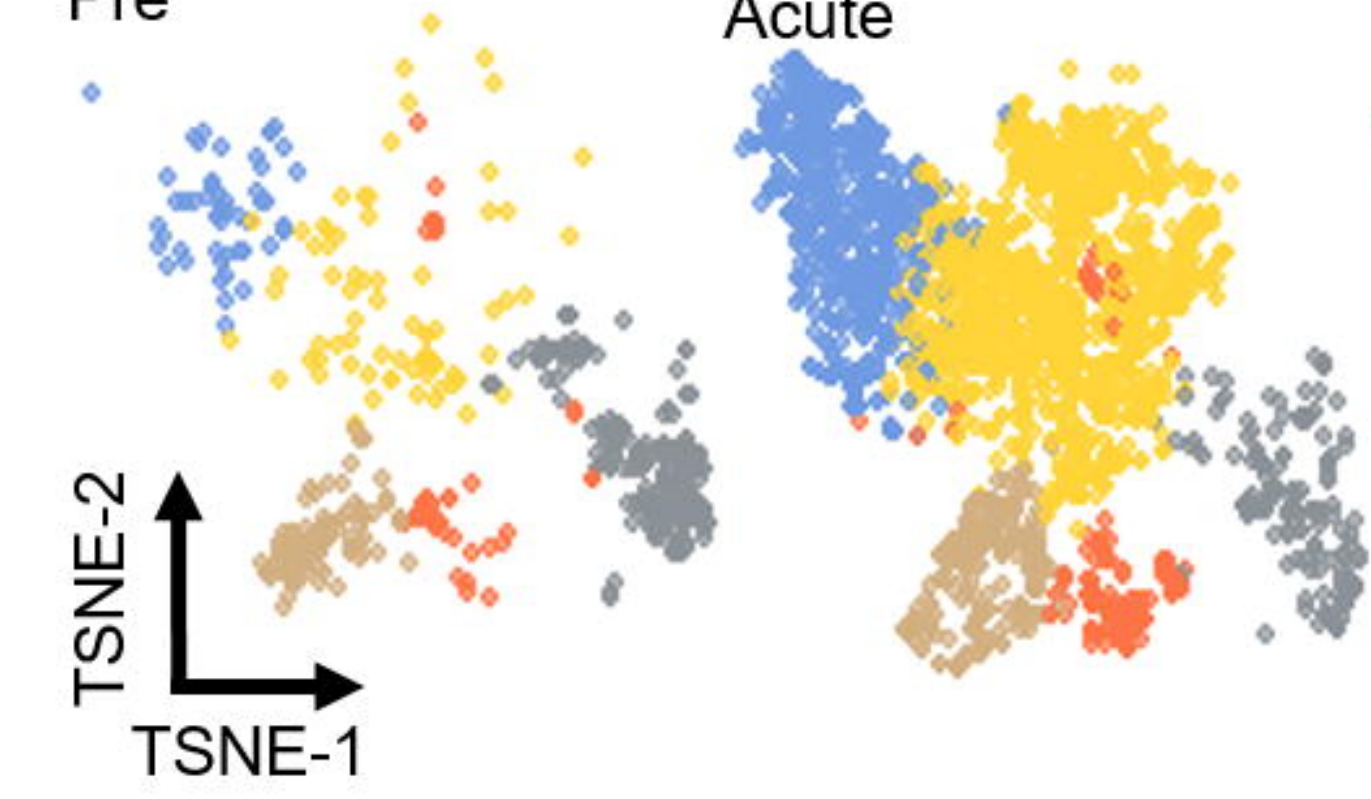

Eosinophil chemotaxis

Regulation of cytokine production Inflammatory response to wounding

Regulation of defense response to bacterium Asthma

Ribosome

Proteasome

Protein export

Proteasome assembly

Structural constituent of ribosome

Other glycan degradation

Ether lipid metabolism

Fatty acid metabolism

Selenoamino acid metabolism

Lysosome

Regulation of autophagy

Negative regulation of ECM organization

Negatixe regulation of Jun kinase activity

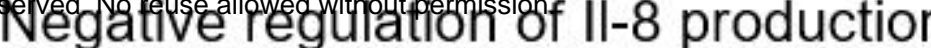

Negative regulation of Th17 immune response

Graft versus host disease

Allograft rejection

Positive regulation of cell-cell adhesion

Negative regulation of vascular permeability

Tissue homeostasis

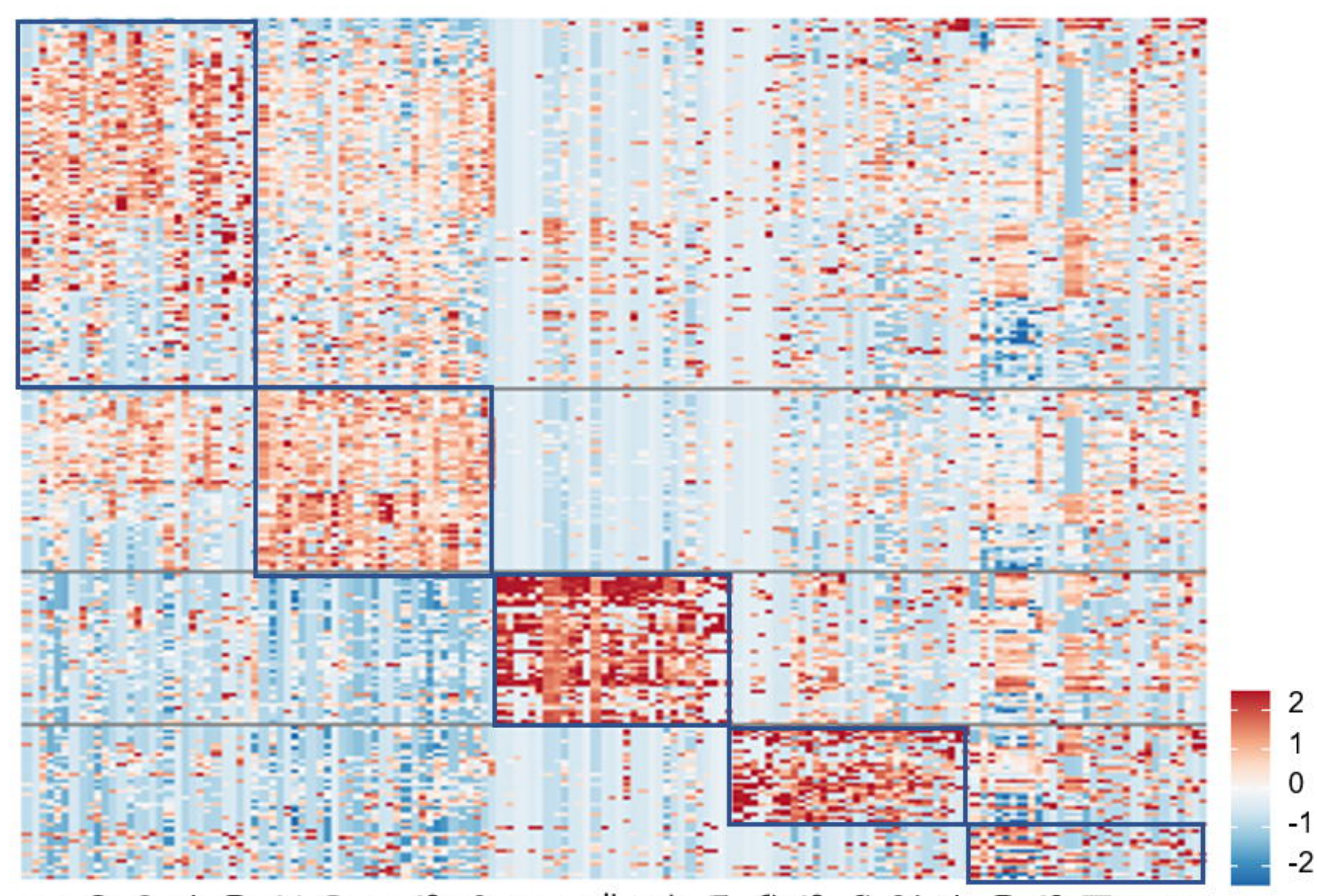

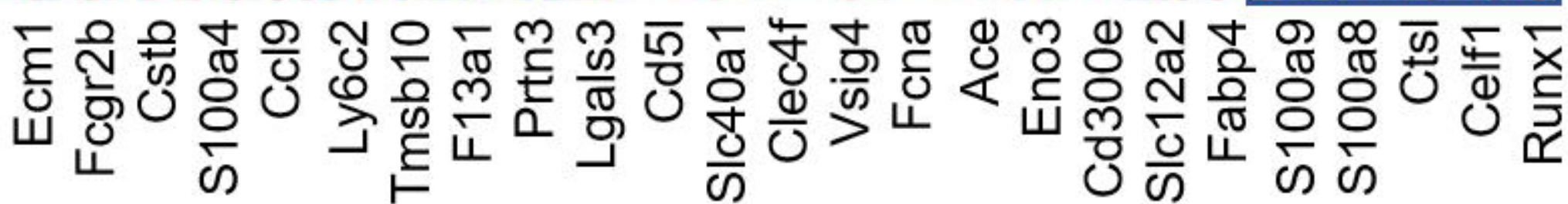

C

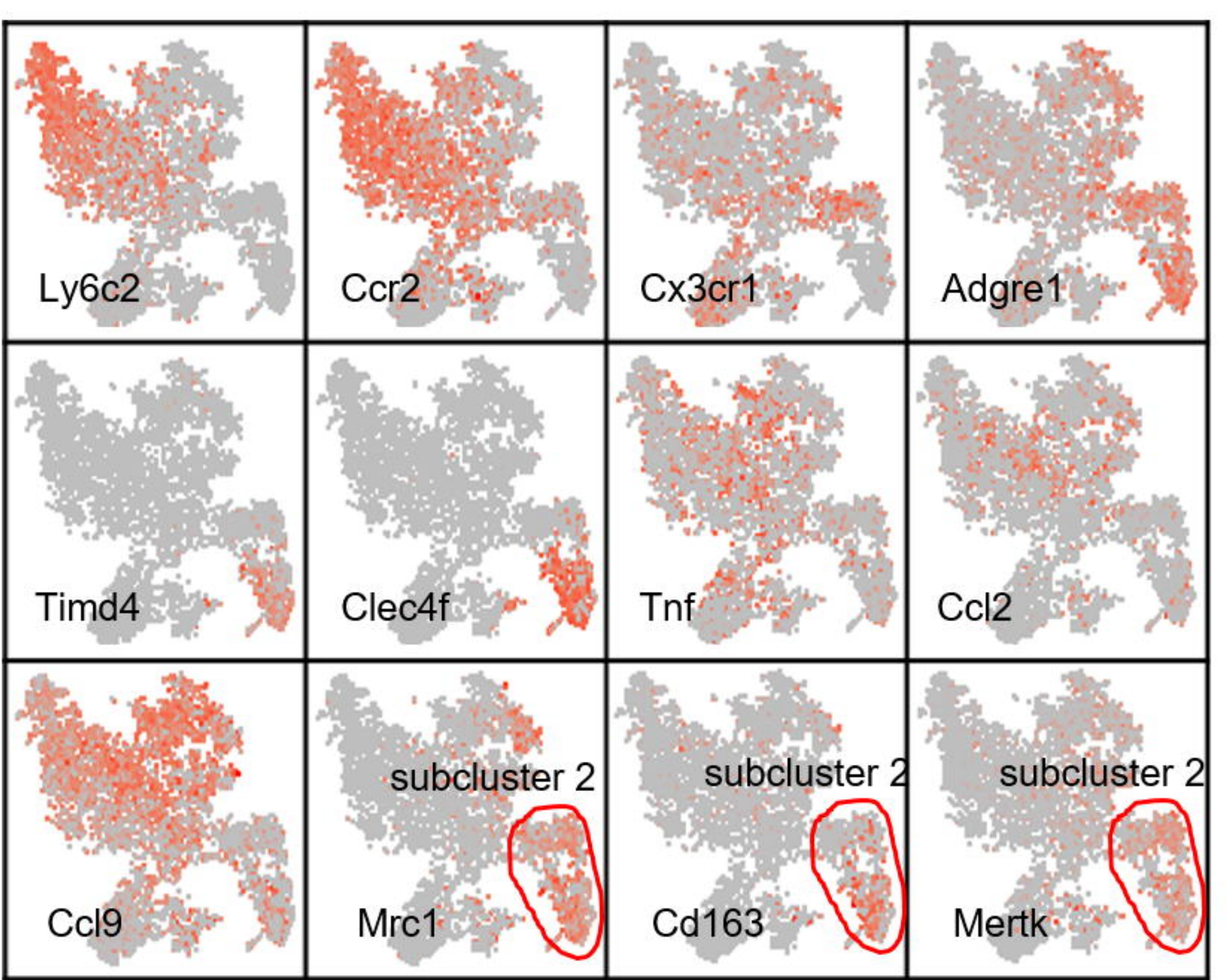

E
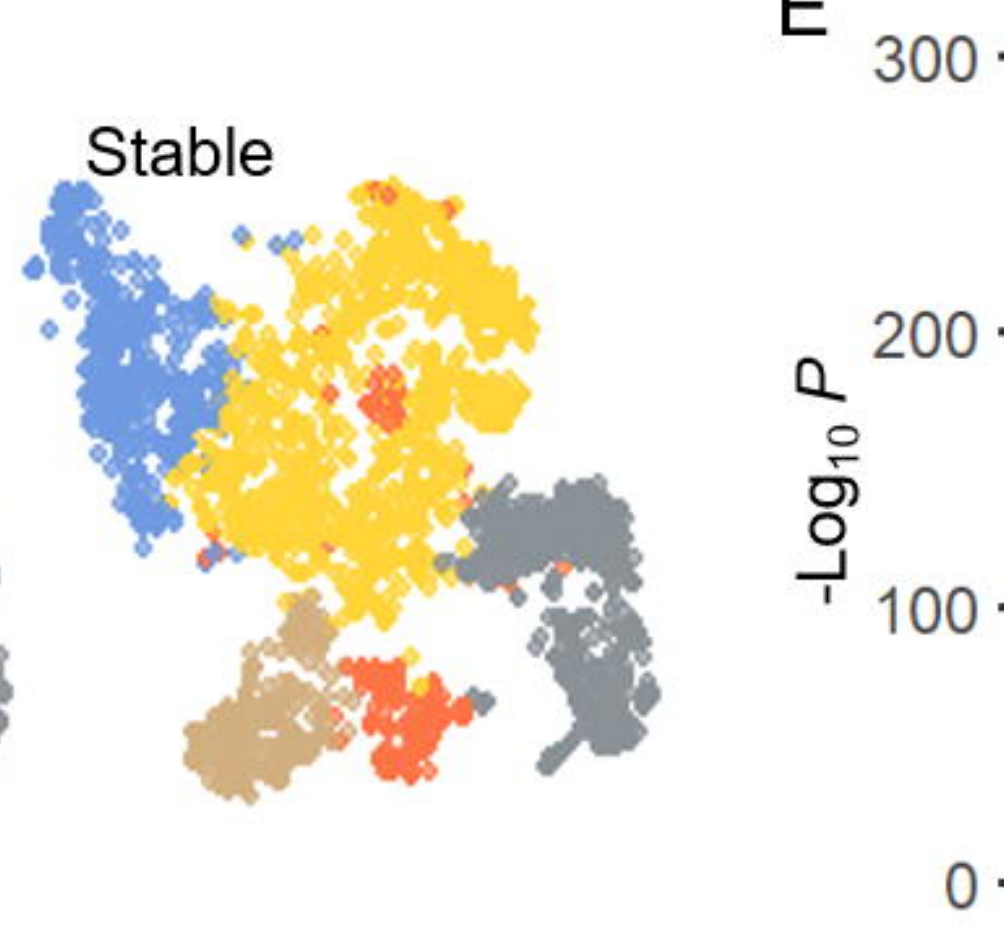

4

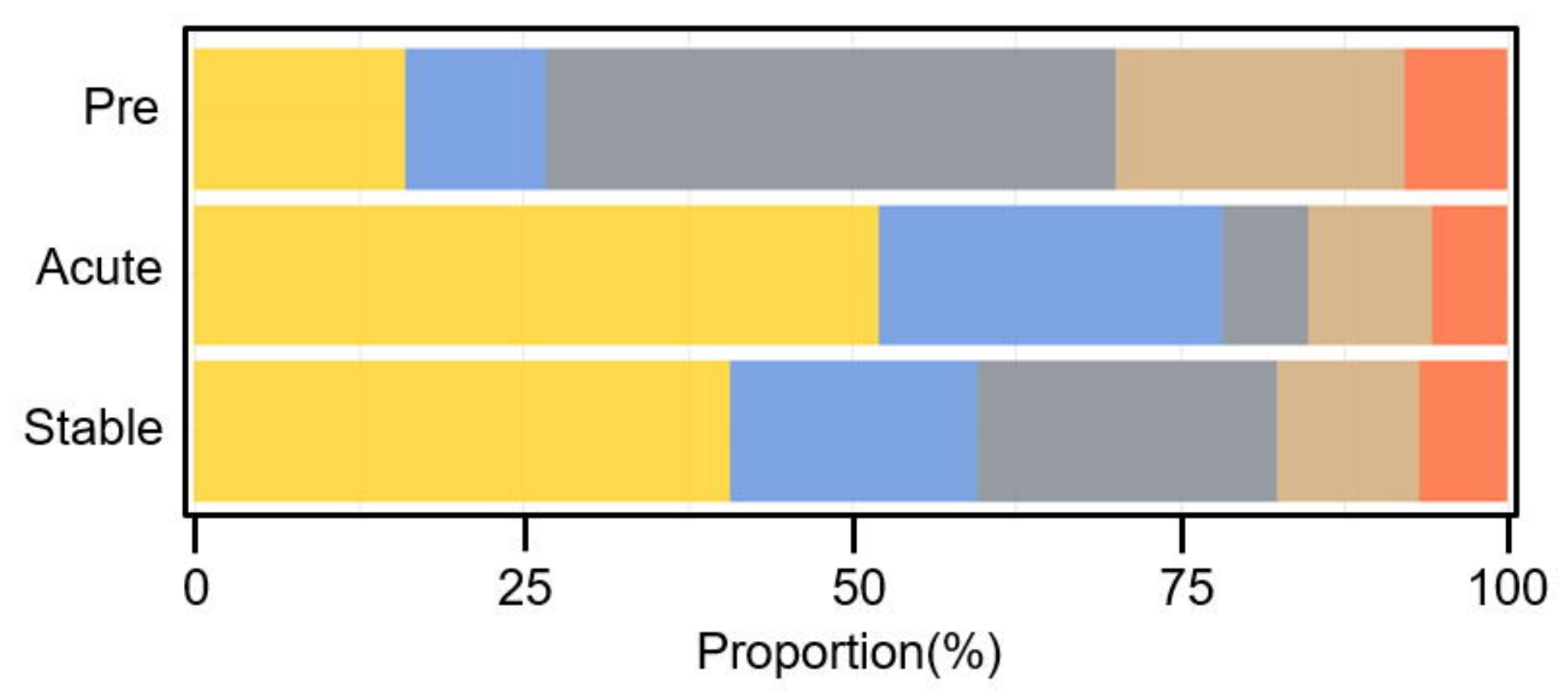

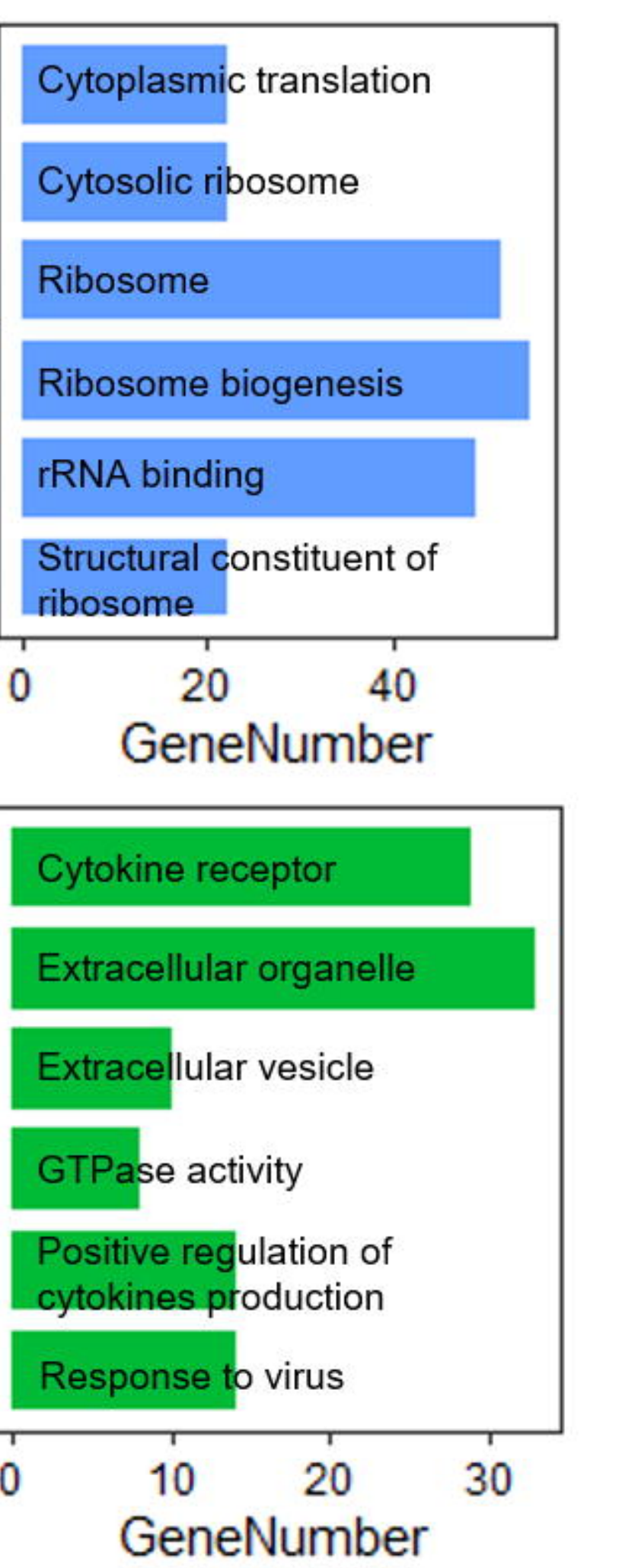


A

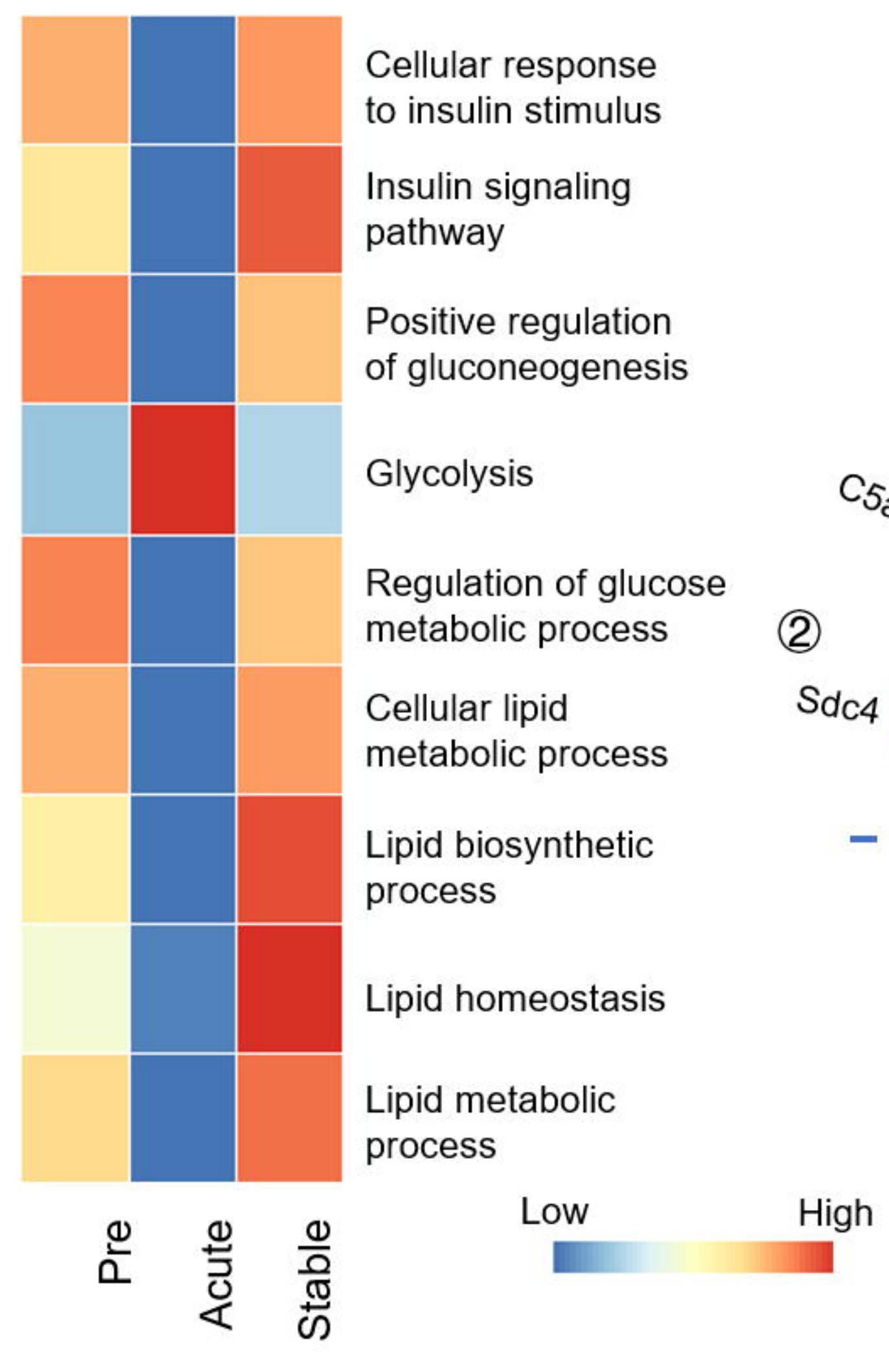

C

Ligands Ligand expression of macrophage activity analysis subcluster 0 / Subcluster 2

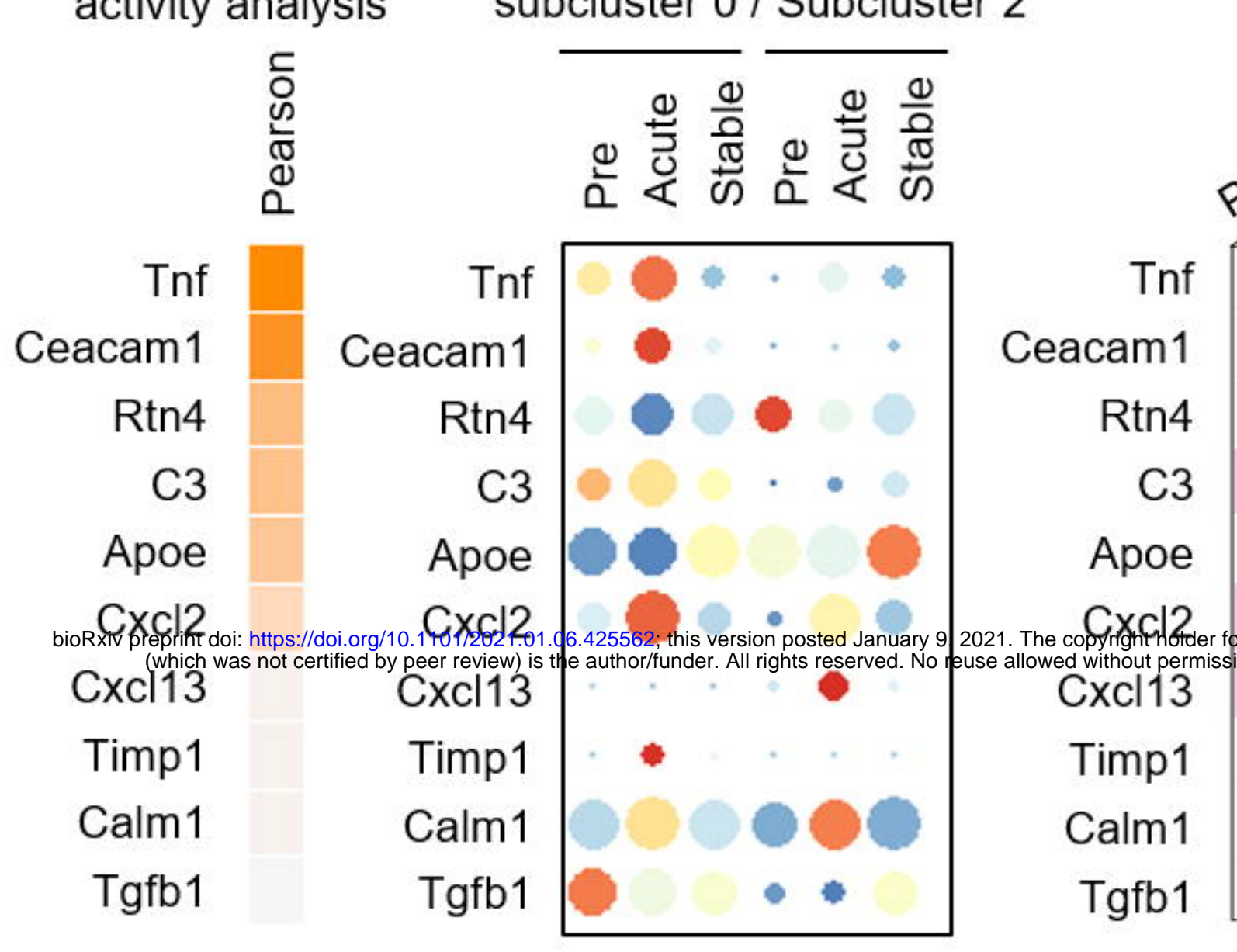

F

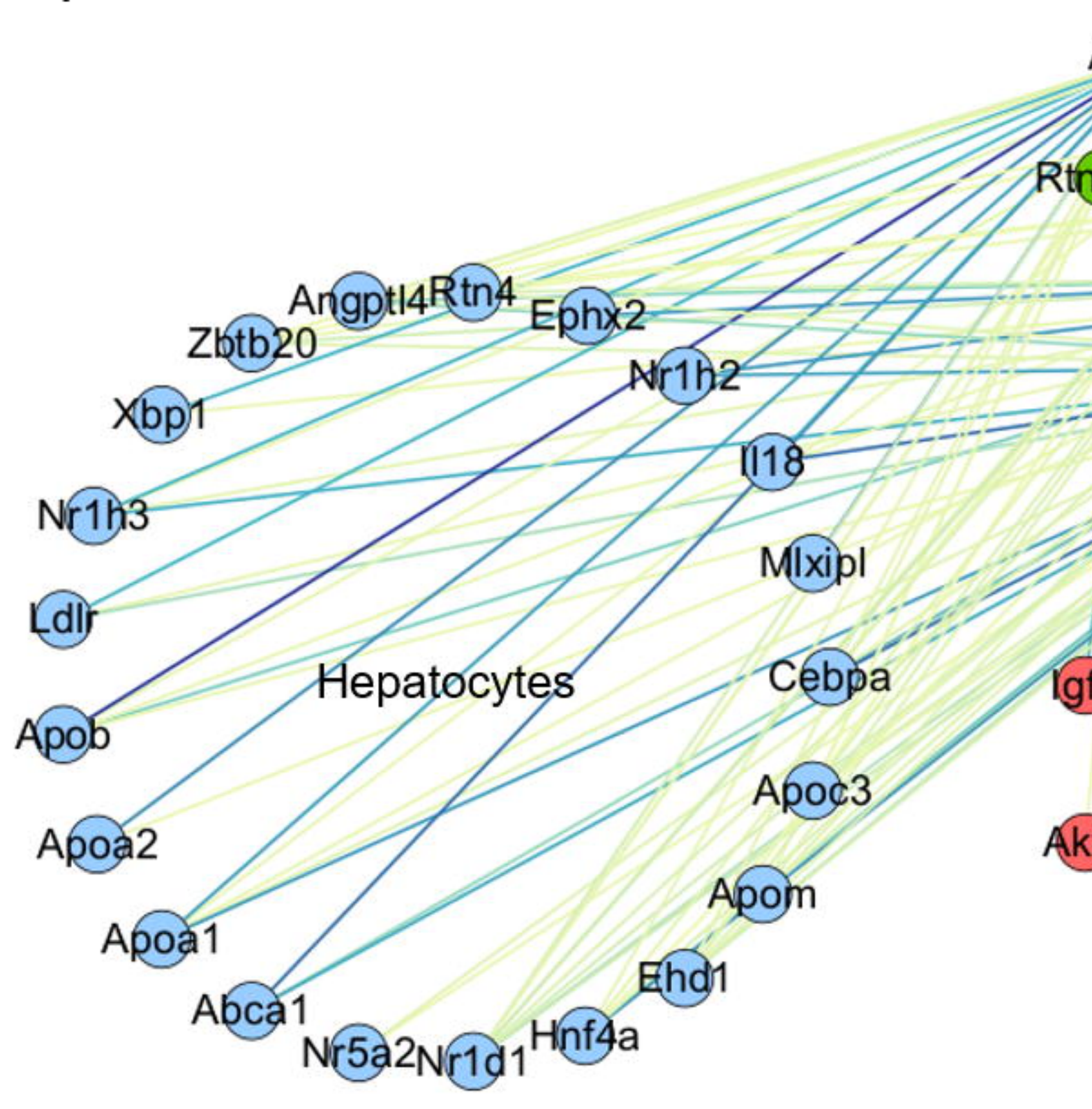

- Receptors

Targets

- Insulin signaling

- Glucose metabolic

- Lipid metabolic $\left\langle g^{2}+1\right.$

$$
\langle 9)^{40}
$$

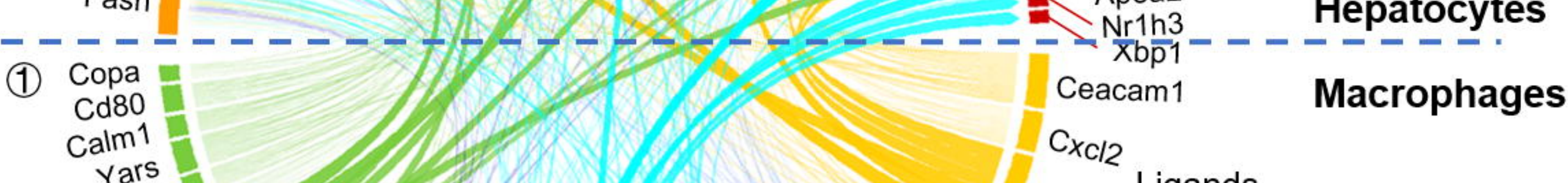

Hepatocytes

\section{Macrophages}

Ligands

- Subcluster 0 High expression

- Subcluster 1 High expression

- Subcluster 2 High expression

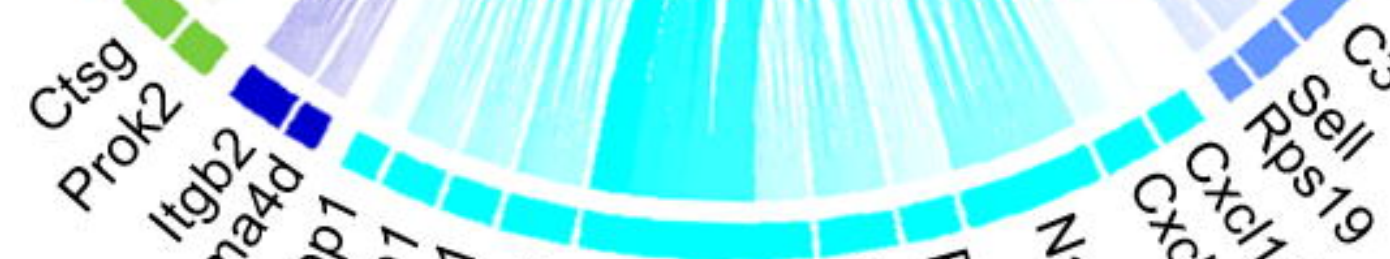

- Subcluster 4 High expression

Common expression

$E$

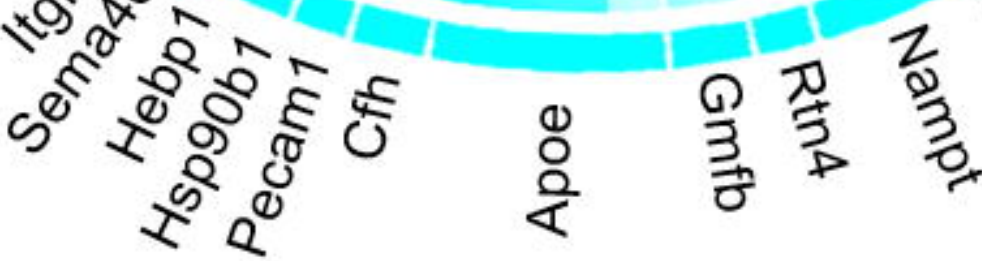

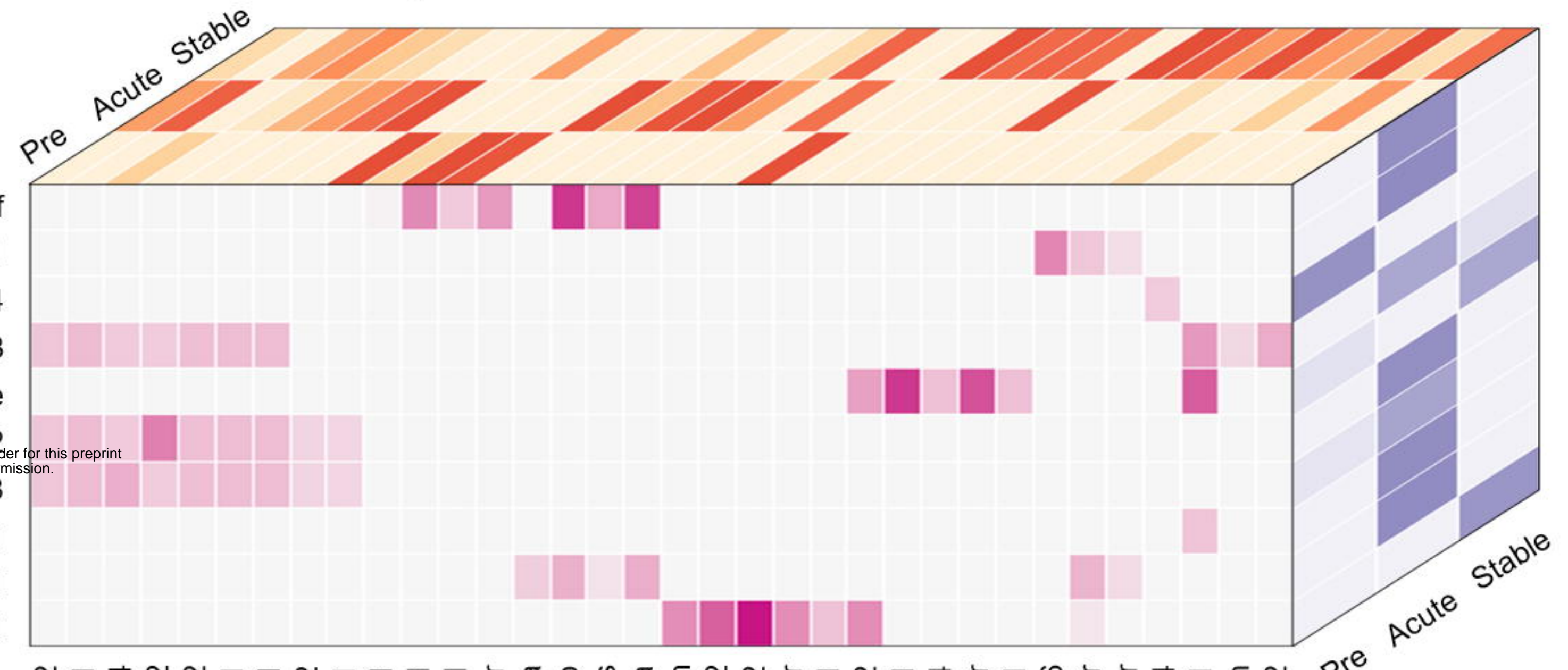

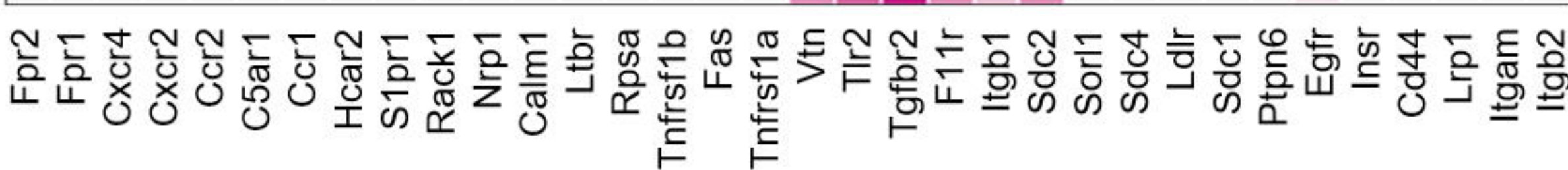

Prior interaction potential

$0.2 \quad 0.4 \quad 0.6$

CxCl2(L) Timpl $(\mathrm{L})$

Apoe(L) $\quad$ Cami(L)

tn4(L) Macrophages Ceacam 1(L)

Q3(2)

$\operatorname{Tg}(\mathrm{b} 1(\mathrm{~L})$

$=\operatorname{Ex} 113(\mathrm{~L}) \mathrm{I}$ If $(\mathrm{L})$

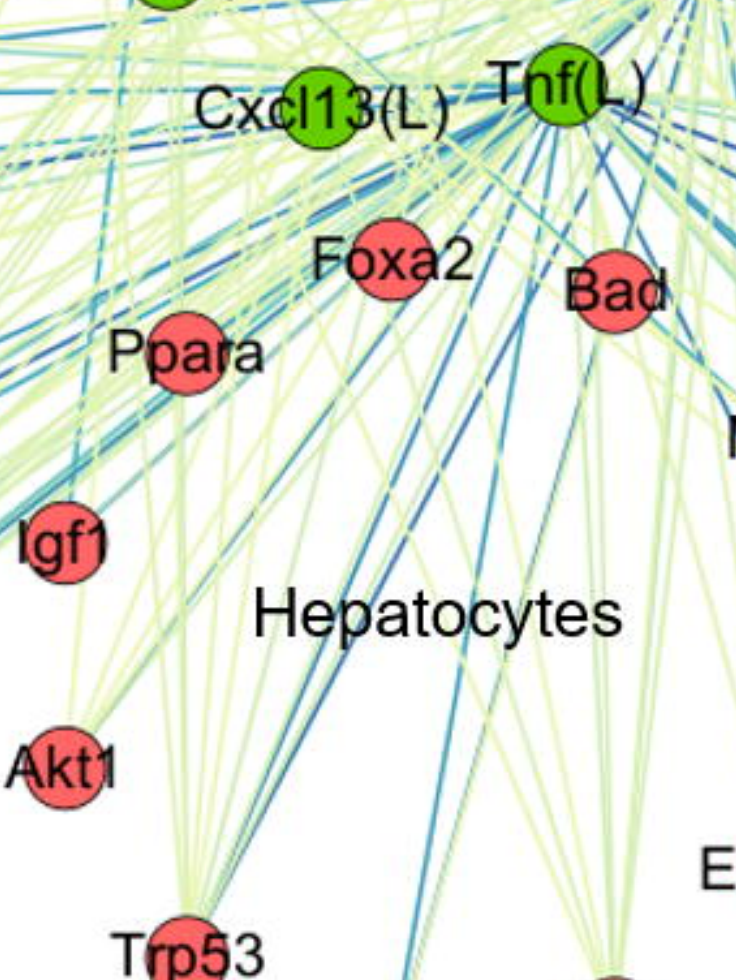

TP53

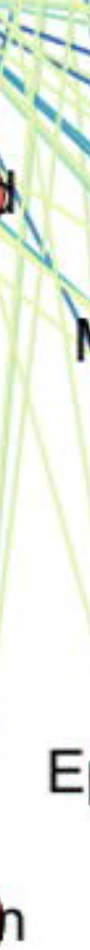

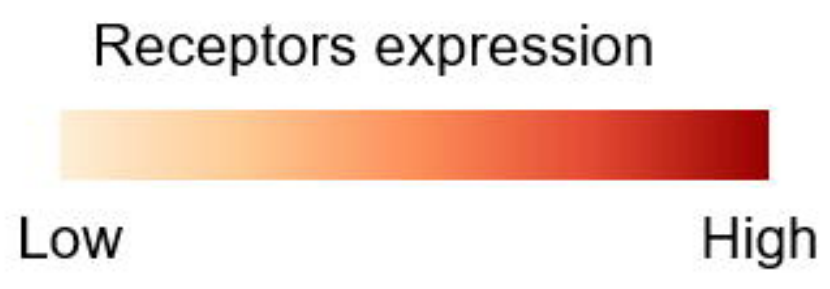

High
Ligands expression

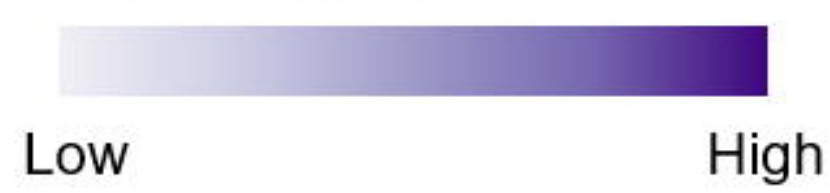

